\title{
Dilepton production and off-shell transport dynamics at SIS energies
}

\author{
E.L. Bratkovskaya, ${ }^{\mathrm{a}, *}$ W. Cassing, ${ }^{\mathrm{b}}$ \\ ${ }^{a}$ Frankfurt Institute for Advanced Studies, Johann Wolfgang Goethe University, \\ Ruth-Moufang-Str. 1, 60438 Frankfurt am Main, Germany \\ ${ }^{\mathrm{b}}$ Institut für Theoretische Physik, Universität Giessen, Heinrich-Buff-Ring 16, \\ D-35392 Giessen, Germany
}

\begin{abstract}
Dilepton production in nucleus-nucleus collisions at 1-2 A.GeV as well as in elementary $p p$ and $p d$ reactions is studied within the microscopic HSD transport approach which includes the off-shell dynamics of vector mesons explicitly. The study addresses additionally the production of $\pi^{0}$ and $\eta$ mesons since their Dalitz decays provide a sizeable contribution to the dilepton invariant mass spectra up to about $0.5 \mathrm{GeV}$. Our transport results agree with the TAPS experimental data on $\pi$ and $\eta$ multiplicities in ${ }^{12} \mathrm{C}+{ }^{12} \mathrm{C}$ collisions from 0.8 to $2 \mathrm{~A} \mathrm{GeV}$. We find that the 'DLSpuzzle' - which stands for a theoretical underestimation of the $e^{+} e^{-}$yield in the mass range from 0.2 to $0.5 \mathrm{GeV}$ in ${ }^{12} \mathrm{C}+{ }^{12} \mathrm{C}$ and ${ }^{40} \mathrm{Ca}+{ }^{40} \mathrm{Ca}$ collisions - may be solved when incorporating a stronger bremsstrahlung contribution in line with recent OBE calculations. Moreover, the HSD results with 'enhanced' bremsstrahlung cross sections agree very well with the HADES experimental data for the dilepton mass spectra for ${ }^{12} \mathrm{C}+{ }^{12} \mathrm{C}$ at 1 and $2 \mathrm{~A} \cdot \mathrm{GeV}$, especially when including a collisional broadening in the vector-meson spectral functions. Detailed predictions for dilepton spectra from $p p$ and $p n / p d$ reactions at $1.25 \mathrm{GeV}, 2.2 \mathrm{GeV}$ and $3.5 \mathrm{GeV}$ are presented which will allow to verify/falsify the larger bremsstrahlung contributions from the experimental side in the near future.
\end{abstract}

Key words: Relativistic heavy-ion collisions, Meson production, Leptons

PACS 25.75.-q, 13.60.Le, 14.60.Cd

\footnotetext{
* corresponding author Email address: Elena.Bratkovskaya@th.physik.uni-frankfurt.de (E.L. Bratkovskaya,).
} 


\section{Introduction}

The theory of quantum-chromo-dynamics (QCD) describes hadrons as many-body bound or resonant states of partonic constituents. While the properties of hadrons are rather well known in free space (embedded in a nonperturbative QCD vacuum) the mass and lifetimes of hadrons in a baryonic and/or mesonic environment are subject of current research in order to achieve a better understanding of the strong interaction and the nature of confinement. In this context the modification of hadron properties in nuclear matter is of fundamental interest (cf. Refs. [1,2,3, 4, $, 6,7,8,9,10,11,12$ for the leading concepts) since QCD sum rules [2,5,13] as well as QCD inspired effective Lagrangian models [1,4,6,7,8,9,10,11,12, predict significant changes e.g. of the vector mesons $(\rho, \omega$ and $\phi)$ with the nuclear density $\rho_{N}$ and/or temperature $T$.

A more direct evidence for the modification of vector mesons has been obtained from the enhanced production of lepton pairs above known sources in nucleus-nucleus collisions at SPS energies [14]15]. As proposed by Li, Ko, and Brown [16] and Ko et al. [17] the observed enhancement in the invariant mass range $0.3 \leq M \leq 0.7$ $\mathrm{GeV}$ might be due to a shift of the $\rho$-meson mass following Brown/Rho scaling [1] or the Hatsuda and Lee sum rule prediction [2]. The microscopic transport studies in Refs. [18, 19,20,2122 for these systems have given support for this interpretation [16, 17, 26, 27]. On the other hand also more conventional approaches that describe a melting of the $\rho$-meson in the medium due to the strong hadronic coupling (along the lines of Refs. $[3,4,6,8,12])$ have been found to be compatible with the early CERES data $8,18,23,24,25]$.

This ambiguous situation has been clarified to some extent by the NA60 Collaboration since the invariant mass spectra for $\mu^{+} \mu^{-}$pairs from In+In collisions at 158 A. GeV clearly favored the 'melting $\rho$ ' scenario [28]. Also the latest data from the CERES Collaboration (with enhanced mass resolution) [29] show a preference for the 'melting $\rho$ ' scenario which is in line with more recent theoretical studies on dilepton production at SPS energies [30,31,32,33,34,35,36,37].

Dileptons have also been measured in heavy-ion collisions at the BEVALAC by the DLS Collaboration [38,39] at incident energies that are two orders-of-magnitude lower than that at the SPS. The first published spectra at $1 \mathrm{~A} \cdot \mathrm{GeV}$ [38] (based on a limited data set) have been consistent with the results from transport model calculations 40,41,42,43 that include $p n$ bremsstrahlung, $\pi^{0}, \eta$ and $\Delta$ Dalitz decay and pion-pion annihilation. However, in 1997 the DLS Collaboration released a new set of data [39] based on the full data sample and an improved analysis, which showed a considerable increase in the dilepton yield: more than a factor of five above the early DLS data [38] and the corresponding theoretical results [40,41,42,43. This discrepancy remained in the transport calculations even after including contributions from the decay of $\rho$ and $\omega$ mesons that are produced directly from nucleon-nucleon and pionnucleon scattering in the early reaction phase [22,44. With an in-medium $\rho$ spectral function, as that used in Ref. [23] for dilepton production from heavy-ion collisions at 
SPS energies, dileptons from the decay of both directly produced $\rho$ 's and pion-pion annihilation have been considered, and a factor of about two enhancement has been obtained in the theoretical studies compared to the case of a free $\rho$-spectral function. Furthermore, in Ref. [45] the alternative scenario of a dropping $\rho$-meson mass and its influence on the properties of the $N(1520)$ resonance has been investigated. Indeed, an incorporation of such medium effects lead to an enhancement of the $\rho$ meson yield, however, was not sufficient to explain the DLS data. Since independent transport calculations by HSD (BUU) and UrQMD underestimated the DLS data for $C+C$ and $C a+C a$ at $1 \mathrm{~A} \mathrm{GeV}$ by roughly the same amount these findings have led to the denotation 'DLS-puzzle' in 1999 [22 44,45] which persists now by about a decade.

As has been shown in 2003 by the transport analysis of the Tübingen group [47] also alternative scenarios for the in-medium modification, i.e. a possible decoherence between the intermediate mesonic states in the vector resonance decay, increases the dilepton yield. However, still the region about $M \simeq 0.3 \mathrm{GeV}$ was underestimated whereas the yield in the vicinity of the $\rho$-meson peak was overestimated (especially for $C+C$ collisons). Thus there is no consistent explanation for the DLS data so far and questions came up about the validity of the DLS data or a possible misinterpretation of the DLS acceptance cuts.

In order to address the in-medium modifications of vector-mesons from the experimental side the HADES spectrometer has been built [61] which allows to study $e^{+} e^{-}$ pair production in a much wider acceptance region for elementary $p p, p d$ reactions as well as $\pi A, p A$ or even $A A$ collisions up to about $8 \mathrm{~A} \cdot \mathrm{GeV}$. During the construction phase of HADES there has been a lot of theoretical activity providing various predictions for these reactions at SIS energies [21,48,49,52,53,54,55,56,57]. Meanwhile the HADES Collaboration has presented first spectra [60,61,62,63] and the question is: what do these data tell us?

The answer to the questions raised above is nontrivial due to the nonequilibrium nature of these reactions and transport models have to be incorporated to disentangle the various sources that contribute to the final dilepton spectra seen experimentally. In this study we aim at contributing to this task employing an up-to-date relativistic transport model (HSD) that incorporates the relevant off-shell dynamics of the vector mesons. Compared to our earlier studies in Refs. [21,23,44,45] a couple of extensions have been implemented such as

- off-shell dynamics for vector mesons - according to Refs. 6465] - and an explicit (nonperturbative) propagation of $\rho, \omega$ and $\phi$ also at SIS energies

- extension of the LUND string model to include 'modified' spectral functions for the resonances in the string decays

- novel cross sections for $\eta$ production in line with more recent experimental data

- novel $N N$ bremsstrahlung cross sections according to recent OBE calculations

- an extended set of spectral functions for the vector mesons $\rho, \omega, \phi$. 
We note that the first off-shell dynamical study for vector mesons and dilepton decays has been presented in Ref. [48] for $p A$ reactions and been picked up later by the Rossendorf group [57] also incorporating the off-shell equations from Refs. [64,65]. The off-shell dynamics is particularly important for resonances with a rather long lifetime in vacuum but strongly decreasing lifetime in the nuclear medium (especially $\omega$ and $\phi$ mesons).

The outline of the paper is as follows: After a brief description of the transport model and its elementary channels in Section 2 we will discuss $\pi^{0}$ and $\eta$ production in elementary as well as $A A$ reactions in comparison to available data (Section 3 ) in order to have independent constraints on the leptonic sources. Section 4 is devoted to dilepton production in $p p$ and $p d$ reactions in confrontation with spectra from the DLS while in Section 5 a comprehensive study of ${ }^{12} \mathrm{C}+{ }^{12} \mathrm{C}$ and ${ }^{40} \mathrm{Ca}+{ }^{40} \mathrm{Ca}$ collisions is performed employing different spectral functions for the vector mesons. In addition we will present predictions for future projects of the HADES Collaboration like $\mathrm{Ar}+\mathrm{KCl}$ at $1.75 \mathrm{~A} \cdot \mathrm{GeV}$ or $p p, p d$ reactions at $1.25 \mathrm{GeV}, 2.2 \mathrm{GeV}$ and $3.5 \mathrm{GeV}$. A summary and discussion closes this work in Section 6.

\section{Description of the model}

Our analysis is carried out within the HSD transport model [20,21,66] - based on covariant self energies for the baryons [58] - that has been used for the description of $p A$ and $A A$ collisions from SIS to RHIC energies. We recall that in the HSD approach nucleons, $\Delta$ 's, $\mathrm{N}^{*}(1440), \mathrm{N}^{*}(1535), \Lambda, \Sigma$ and $\Sigma^{*}$ hyperons, $\Xi$ 's, $\Xi^{*}$ 's and $\Omega$ 's as well as their antiparticles are included on the baryonic side whereas the $0^{-}$and $1^{-}$octet states are incorporated in the mesonic sector. Inelastic baryon-baryon (and meson-baryon) collisions with energies above $\sqrt{s}_{t h} \simeq 2.6 \mathrm{GeV}$ (and $\sqrt{s}_{t h} \simeq 2.3 \mathrm{GeV}$ ) are described by the FRITIOF string model [67] whereas low energy hadron-hadron collisions are modeled in line with experimental cross sections.

The dilepton production by a (baryonic or mesonic) resonance $R$ decay can be schematically presented in the following way:

$$
\begin{aligned}
B B \rightarrow & R X \\
m B \rightarrow & R X \\
& R \rightarrow e^{+} e^{-} X, \\
& R \rightarrow m X, m \rightarrow e^{+} e^{-} X \\
& R \rightarrow R^{\prime} X, R^{\prime} \rightarrow e^{+} e^{-} X
\end{aligned}
$$

i.e. in a first step a resonance $R$ might be produced in baryon-baryon $(B B)$ or mesonbaryon $(m B)$ collisions (1), (2). Then this resonance can couple to dileptons directly (3) (e.g., Dalitz decay of the $\Delta$ resonance: $\Delta \rightarrow e^{+} e^{-} N$ ) or decays to a meson $m$ 
(+ baryon) or in (44) produce dileptons via direct decays $(\rho, \omega)$ or Dalitz decays $\left(\pi^{0}, \eta, \omega\right)$. The resonance $R$ might also decay into another resonance $R^{\prime}$ (5) which later produces dileptons via Dalitz decay. Note, that in the combined model the final particles - which couple to dileptons - can be produced also via non-resonant mechanisms, i.e. 'background' channels at low and intermediate energies or string decay at high energies [59].

The electromagnetic part of all conventional dilepton sources $-\pi^{0}, \eta, \omega$ Dalitz decays, direct decay of vector mesons $\rho, \omega$ and $\phi$ - are treated as described in detail in Ref. [68]- where dilepton production in $p p$ and $p d$ reactions has been studied. Modifications - relative to Ref. 68] - are related to the Dalitz decay of baryonic resonances and especially the strength of the $p p$ and $p n$ bremsstrahlung since calculations by Kaptari and Kämpfer in 2006 [69] indicated that the latter channels might have been severely underestimated in our previous studies on dilepton production at SIS energies. In detail:

- For the Dalitz decays of the baryonic resonances we adopt the parametrizations from Ernst et al. 22] (eqs. (9) to (13)) which are also incorporated in the PLUTO simulation program of the HADES Collaboration [70].

- For the bremsstrahlung channels in $p p$ and $p n$ reactions we adjust our previous expressions in order to match the recent results from the OBE model calculations in Ref. 69] (cf. Section 2.5).

\subsection{Vector-meson spectral functions}

In order to explore the influence of in-medium effects on the vector-meson spectral functions we incorporate the effect of collisional broadening (as in Refs. [45|72]), i.e. the vector meson width has been implemented as:

$$
\Gamma_{V}^{*}\left(M,|\vec{p}|, \rho_{N}\right)=\Gamma_{V}(M)+\Gamma_{\text {coll }}\left(M,|\vec{p}|, \rho_{N}\right) .
$$

Here $\Gamma_{V}(M)$ is the total width of the vector mesons $(V=\rho, \omega)$ in the vacuum. For the $\rho$ meson we use

$$
\begin{gathered}
\Gamma_{\rho}(M) \simeq \Gamma_{\rho \rightarrow \pi \pi}(M)=\Gamma_{0}\left(\frac{M_{0}}{M}\right)^{2}\left(\frac{q}{q_{0}}\right)^{3} F(M) \\
q=\frac{\left(M^{2}-4 m_{\pi}^{2}\right)^{1 / 2}}{2}, \quad q_{0}=\frac{\left(M_{0}^{2}-4 m_{\pi}^{2}\right)^{1 / 2}}{2} .
\end{gathered}
$$

In (7) $M_{0}$ is the vacuum pole mass of the vector meson spectral function, $F(M)$ is a formfactor taken from Ref. [8] as 


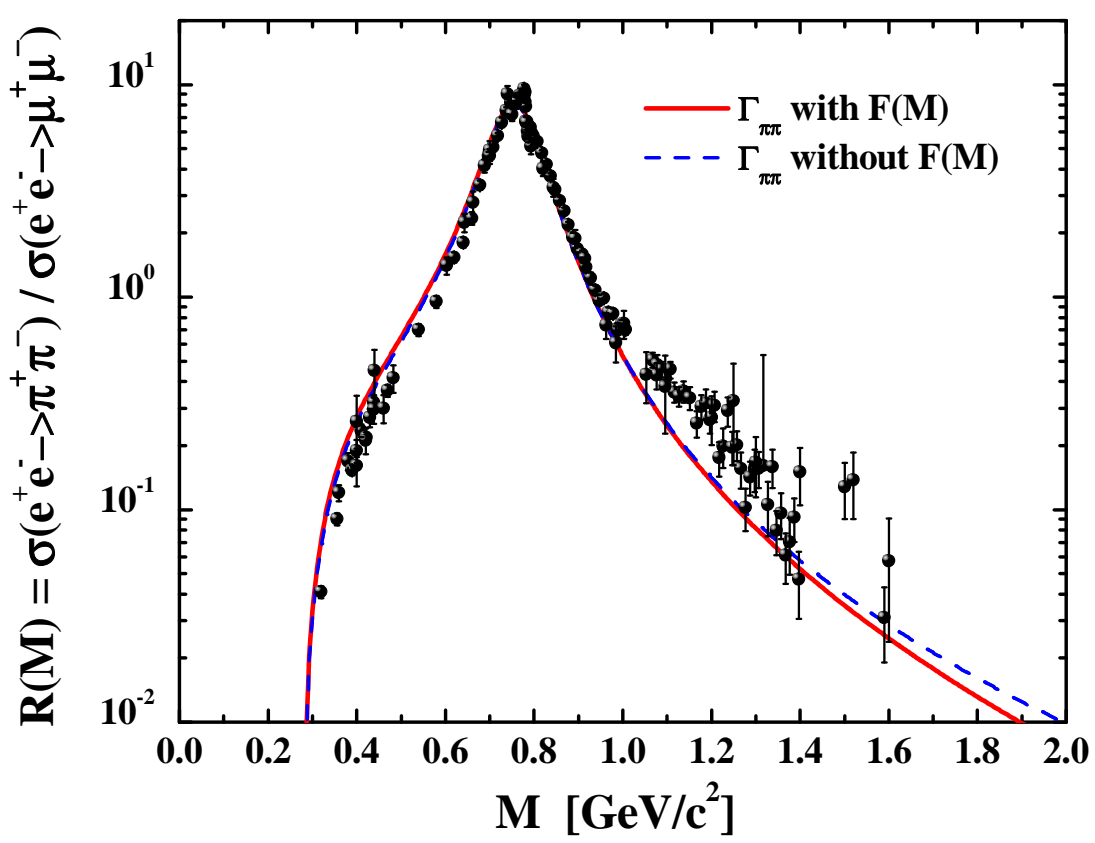

Fig. 1. The ratio $R(M)=\sigma\left(e^{+} e^{-} \rightarrow \pi^{+} \pi^{-}\right) / \sigma\left(e^{+} e^{-} \rightarrow \mu^{+} \mu^{-}\right)$. The solid (red) line corresponds to the result with the formfactor $F(M)$ (8) while the dashed (blue) line is without $F(M)$. The compilation of the experimental data is taken from Ref. [73].

$$
F(M)=\left(\frac{2 \Lambda^{2}+M_{0}^{2}}{2 \Lambda^{2}+M^{2}}\right)^{2}
$$

with a cut-off parameter $\Lambda=3.1 \mathrm{GeV}$. This formfactor was introduced in Ref. [8] in order to describe the $e^{+} e^{-}$experimental data with better accuracy. In Fig. 10 we show the ratio $R(M)=\sigma\left(e^{+} e^{-} \rightarrow \pi^{+} \pi^{-}\right) / \sigma\left(e^{+} e^{-} \rightarrow \mu^{+} \mu^{-}\right)$which is proportional to the pion formfactor $F_{\pi}(M)$ except for a kinematical factor. The solid line corresponds to the result with the formfactor $F(M)$ (8) while the dashed line is without $F(M)$. The compilation of the experimental data is taken from Ref. [73]. As seen from Fig. 1 the effect of the formfactor $F(M)$ (8) is visible only at high $M$.

For the $\omega$ meson a constant total vacuum width is used: $\Gamma_{\omega} \equiv \Gamma_{\omega}\left(M_{0}\right)$, since the $\omega$ is a narrow resonance in vacuum.

The collisional width in (6) is approximated as

$$
\Gamma_{\text {coll }}\left(M,|\vec{p}|, \rho_{N}\right)=\gamma \rho_{N}<v \sigma_{V N}^{t o t}>\approx \alpha_{\text {coll }} \frac{\rho_{N}}{\rho_{0}}
$$

Here $v=|\vec{p}| / E ; \vec{p}, E$ are the velocity, 3-momentum and energy of the vector meson in the rest frame of the nucleon current and $\gamma^{2}=1 /\left(1-v^{2}\right)$. Furthermore, $\rho_{N}$ is the nuclear density and $\sigma_{V N}^{\text {tot }}$ the meson-nucleon total cross section.

In order to simplify the actual calculations for dilepton production the coefficient $\alpha_{\text {coll }}$ has been extracted in the HSD transport calculations from the vector-meson 
collision rate in $C+C$ and $C a+C a$ reactions (at 1 and $2 \mathrm{~A} \cdot \mathrm{GeV}$ ) as a function of the density $\rho_{N}$. In case of the $\rho$ meson the collision rate is dominated by the absorption channels $\rho N \rightarrow \pi N$ or $\rho N \rightarrow \Delta \pi \rightarrow \pi \pi N$ at SIS energies. Also the reactions $\rho+\pi \leftrightarrow a_{1}$ are incorporated but of minor importance here. The numerical results for $\Gamma_{\text {coll }}\left(\rho_{N}\right)$ then have been divided by $\rho_{N} / \rho_{0}$ to fix the coefficient $\alpha_{\text {coll }}$ in (9). We obtain $\alpha_{\text {coll }} \approx 150 \mathrm{MeV}$ for the $\rho$ and $\alpha_{\text {coll }} \approx 70 \mathrm{MeV}$ for $\omega$ mesons which are consistent with Ref. [74]. In this way the average effects of collisional broadening are incorporated in accordance with the transport calculations and allow for an explicit representation of the vector-meson spectral functions versus the nuclear density (see below). We point out, however, that only the average width of the vector mesons is consistent with the dynamical evolution of these particles in the transport calculations and not the specific shape of their spectral functions.

In order to explore the observable consequences of vector meson mass shifts at finite nuclear density - as indicated by the CBELSA-TAPS data [75] for the $\omega$ meson the in-medium vector meson pole masses are modeled (optionally) according to the Hatsuda and Lee [2] or Brown/Rho scaling [1] as

$$
M_{0}^{*}\left(\rho_{N}\right)=\frac{M_{0}}{\left(1+\alpha \rho_{N} / \rho_{0}\right)},
$$

where $\rho_{N}$ is the nuclear density at the resonance decay position $\vec{r} ; \rho_{0}=0.16 \mathrm{fm}^{-3}$ is the normal nuclear density and $\alpha \simeq 0.16$ for the $\rho$ and $\alpha \simeq 0.12$ for the $\omega$ meson [74. The parametrization (10) may be employed also at much higher collision energies (e.g. FAIR and SPS) and one does not have to introduce a cut-off density in order to avoid negative pole masses. Note that (10) is uniquely fixed by the 'customary' expression $M_{0}^{*}\left(\rho_{N}\right) \approx M_{0}\left(1-\alpha \rho_{N} / \rho_{0}\right)$ in the low density regime.

The spectral function of the vector meson $V$ for the mass $M$ at baryon density $\rho_{N}$ is taken in the Breit-Wigner form:

$$
A_{V}\left(M, \rho_{N}\right)=C_{1} \cdot \frac{2}{\pi} \frac{M^{2} \Gamma_{V}^{*}\left(M, \rho_{N}\right)}{\left(M^{2}-M_{0}^{*}\left(\rho_{N}\right)\right)^{2}+\left(M \Gamma_{V}^{*}\left(M, \rho_{N}\right)\right)^{2}} .
$$

The factor $C_{1}$ is fixed by the normalization condition for arbitrary $\rho_{N}$ :

$$
\int_{M_{\min }}^{M_{\text {lim }}} A_{V}\left(M, \rho_{N}\right) d M=1
$$

where $M_{\text {lim }}=2 \mathrm{GeV}$ is chosen as an upper limit for the numerical integration. The lower limit for the vacuum spectral function corresponds to the two-pion decay, $M_{\text {min }}=2 m_{\pi}$, whereas for the in-medium collisional broadening case $M_{\min }=2 m_{e} \rightarrow$ 0 with $m_{e}$ denoting the electron mass. $M_{0}^{*}$ is the pole mass of the vector meson 

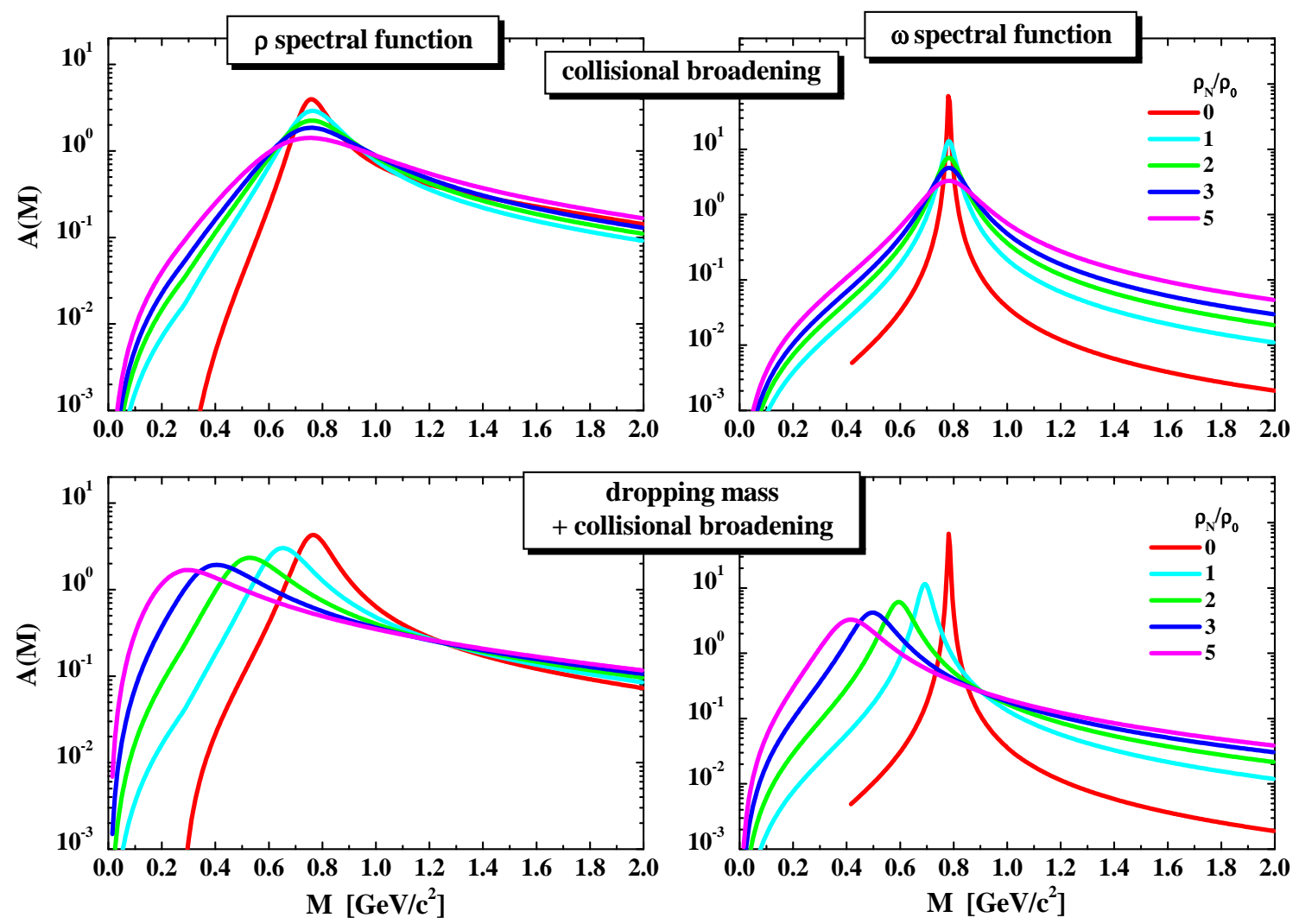

Fig. 2. The spectral functions for the $\rho$ and $\omega$ meson in the case of the 'collisional broadening' scenario (upper part) and the 'dropping mass + collisional broadening' scenario (lower part) for nuclear densities of $0,1,2,3,5 \times \rho_{0}$ as employed in the transport calculations (see text for details).

spectral function which is $M_{0}^{*}\left(\rho_{N}=0\right)=M_{0}$ in vacuum, however, may be shifted in the medium for the dropping mass scenario according to Eq. (10).

The resulting spectral functions for the $\rho$ and $\omega$ meson are displayed in Fig. 2 for the case of 'collisional broadening' (upper part) as well as for the 'dropping mass + collisional broadening' scenario (lower part) for densities of $0,1,2,3,5 \times \rho_{0}$. Note that in vacuum the hadronic widths vanish for the $\rho$ below the two-pion mass and for the $\omega$ below the three-pion mass. With increasing nuclear density $\rho_{N}$ elastic and inleastic interactions of the vector mesons shift strength to low invariant masses. In the 'collisional broadening' scenario we find a dominant enhancement of strength below the pole mass for the $\rho$ meson while the $\omega$ meson spectral function is drastically enhanced in the low- and high-mass region with density (on expense of the polemass regime). In the 'dropping mass + collisional broadening' scenario both vector mesons dominantly show a shift of strength to low invariant masses with increasing $\rho_{N}$. Qualitatively similar pictures are obtained for the $\phi$ meson but quantitatively smaller effects are seen due to the lower effect of mass shifts and a substantially reduced $\phi N$ cross section which is a consequence of the $s \bar{s}$ substructure of the $\phi$ meson. Since the $\phi$ dynamics turn out to be of minor importance for the dilepton 
spectra to be discussed below we discard an explicit representation.

The 'family' of spectral functions shown in Fig. 1 allows for a sufficient flexibility with respect to the possible scenarios outlined above. A comparison to dilepton data is expected to provide further constraints on the possible realizations.

\subsection{Off-shell propagation}

The underlying concept of all 'on-shell' semi-classical transport models (VUU, BUU, QMD, UrQMD etc.) is based on a Monte-Carlo solution of the transport equations for the phase-space densities of hadrons which are obtained in first order gradient expansion of the Wigner transformed Kadanoff-Baym equations using the quasiparticle approximation (QPA). The latter (QPA) assumes that the particles have narrow (i.e. $\delta$-like) spectral functions which don't change their shape during the propagation. This concept works sufficiently well for weakly interacting particles with a very long lifetime but becomes inadequate for short-lived (broad) resonance states of high collision rate.

A first attempt to dynamically account for in-medium effects of vector mesons (i.e. a broadening of their spectral function) using the standard on-shell transport model showed a couple of severe problems (cf. the study by Effenberger et al. in Ref. [93]). The most serious one is that e.g. a $\rho$ meson with a broad spectral function in the medium does not regain its vacuum spectral shape when propagating out of the medium 93. The reason is that dynamical changes of the spectral function during propagation are only included by explicit collisions with other particles in conventional on-shell models (cf. the discussion in context with Fig. 2 below).

In order to allow for a proper phase-space description of broad resonances with varying spectral functions the off-shell transport dynamics (OSTD) has been developed in Refs. [64,65]. The main idea of the OSTD is to discard the quasiparticle approximation in the first order gradient expansion of the Wigner transformed Kadanoff-Baym equations. This leads to generalized transport equations which contain an additional 'backflow' term that vanishes in the on-shell QPA, however, survives in the off-shell case and becomes very important for the dynamics of broad resonances [64,65] (see below). A first application of the OSTD to dilepton production has been presented already in 2001 by one of the authors [48. It has been shown that using OSTD the broad spectral function of vector mesons merges to the vacuum shape by propagation out of the dense medium.

Here we briefly describe the implementation of OSTD in the HSD model: A vector meson $V$ - created at some space-time point $x$ at density $\rho_{N}$ - is distributed in mass according to its spectral function $A_{V}\left(M, \rho_{N}\right)$ (and the available phase space). While propagating through the nuclear medium the total width of the vector meson

$\Gamma_{V}^{*}\left(M, \rho_{N}\right)$ (6) changes dynamical and its spectral function (11) is also modified 
according to the real part of the vector meson self energy $R e \Sigma^{r e t}$ as well as by the imaginary part of the self energy $\left(\Gamma_{V}^{*} \simeq-\operatorname{Im} \Sigma^{r e t} / M\right)$ following

$$
A_{V}\left(M, \rho_{N}\right)=C_{1} \cdot \frac{2}{\pi} \frac{M^{2} \Gamma_{V}^{*}}{\left(M^{2}-M_{0}^{2}-R e \Sigma^{r e t}\right)^{2}+\left(M \Gamma_{V}^{*}\right)^{2}}
$$

which is the in-medium form for a scalar boson spectral function. The spectral function has to change during propagation and to merge the vacuum spectral function when propagating out of the medium.

In the OSTD the general off-shell equations of motion for test particles with momentum $\vec{P}_{i}$, energy $\varepsilon_{i}$ at position $\vec{X}_{i}$ read [64,65]

$$
\begin{aligned}
& \frac{d \vec{X}_{i}}{d t}=\frac{1}{1-C_{(i)}} \frac{1}{2 \varepsilon_{i}}\left[2 \vec{P}_{i}+\vec{\nabla}_{P_{i}} \operatorname{Re} \Sigma_{(i)}^{r e t}+\frac{\varepsilon_{i}^{2}-\vec{P}_{i}^{2}-M_{0}^{2}-\operatorname{Re} \Sigma_{(i)}^{r e t}}{\tilde{\Gamma}_{(i)}} \vec{\nabla}_{P_{i}} \tilde{\Gamma}_{(i)}\right] \\
& \frac{d \vec{P}_{i}}{d t}=-\frac{1}{1-C_{(i)}} \frac{1}{2 \varepsilon_{i}}\left[\vec{\nabla}_{X_{i}} \operatorname{Re} \Sigma_{i}^{r e t}+\frac{\varepsilon_{i}^{2}-\vec{P}_{i}^{2}-M_{0}^{2}-\operatorname{Re} \Sigma_{(i)}^{r e t}}{\tilde{\Gamma}_{(i)}} \vec{\nabla}_{X_{i}} \tilde{\Gamma}_{(i)}\right] \\
& \frac{d \varepsilon_{i}}{d t}=\frac{1}{1-C_{(i)}} \frac{1}{2 \varepsilon_{i}}\left[\frac{\partial R e \Sigma_{(i)}^{r e t}}{\partial t}+\frac{\varepsilon_{i}^{2}-\vec{P}_{i}^{2}-M_{0}^{2}-\operatorname{Re} \Sigma_{(i)}^{r e t}}{\partial \tilde{\Gamma}_{(i)}} \frac{\tilde{\Gamma}_{(i)}}{\partial t}\right]
\end{aligned}
$$

where the notation $F_{(i)}$ implies that the function is taken at the coordinates of the test particle, i.e. $F_{(i)} \equiv F\left(t, \vec{X}_{i}(t), \vec{P}_{i}(t), \varepsilon_{i}(t)\right)$. In Eqs. (14)-(16) Re $\sum^{\text {ret }}$ denotes the real part of the retarded self energy while $\tilde{\Gamma}=-\operatorname{Im} \Sigma^{\text {ret }}$ stands for the (negative) imaginary part in short-hand notation. Apart from the propagation in the real potential $\sim R e \Sigma^{r e t} / 2 \varepsilon$ the equations (14) - (16) include the dynamical changes due to the imaginary part of the self energy $\operatorname{Im} \Sigma^{r e t} \sim-M \Gamma_{V}^{*}$ with $\Gamma_{V}^{*}$ from (6) $)$.

In Eqs. (14) - (16) a common factor $\left(1-C_{(i)}\right)^{-1}$ appears, which includes the energy derivatives of the retarded selfenergy,

$$
C_{(i)}=\frac{1}{2 \varepsilon_{i}}\left[\frac{\partial R e \Sigma_{(i)}^{r e t}}{\partial \epsilon_{i}}+\frac{\varepsilon_{i}^{2}-\vec{P}_{i}^{2}-M_{0}^{2}-R e \Sigma_{(i)}^{r e t}}{\tilde{\Gamma}_{(i)}} \frac{\partial \tilde{\Gamma}_{(i)}}{\partial \epsilon_{i}}\right]
$$

This common factor leads to a rescaling of the 'eigentime' of particle $i$ but does not change the trajectories as demonstrated in Fig. 3 of Ref. [65]). According to the model studies in 65] this prefactor is negative for invariant masses $M$ below the pole mass $M_{0}$ and positive above which implies that the factor $1 /\left(1-C_{i}\right)$ is less than 1 for $M<$ $M_{0}$ and larger than 1 for $M>M_{0}$ (as stated in [65]). This yields a time dilatation for masses $M<M_{0}$ in their phase-space propagation. In order to examine the possible effect on dilepton spectra we have repeated the model calculations discussed in Fig. 
3 of [65] including additionally the decay of each 'testparticle' according to the width $\tilde{\Gamma} /\left(2 M_{i}\right)$ in the eigen frame of the particle. Furthermore, Eqs. (22),(23) in Section 2.5 of the present manuscript have been adopted to calculate the influence on dilepton spectra by integrating the differential decay rate in time. As initial condition 'broad vector quasiparticles' close to the center of a 'nucleus' have been assumed whereas the other parameters have been taken the same as in Fig. 3 of [65]. The resulting 'dilepton spectra' show a slight enhancement with respect to the reference calculation for masses $M<M_{0}$ when including the correction factor. This results from a slightly larger 'lifetime' of the particles for $M<M_{0}$ - when including the rescaling of the 'lifetime' by the correction factor - and leads to a longer dilepton radiation time. The small differences obtained can be traced back to the size of the correction factor for $M<M_{0}$ for the model case in Ref. [65] which is less than $12 \%$. Accordingly we find the 'enhancement' in the dilepton yield to be less than $12 \%$ for the model scenario, too. Since the explicit energy dependence of the retarded selfenergy is highly model dependent we will discard the terms (17) in the following study, i.e. assume $C_{(i)}=0$.

The interpretation of the equations of motion (14) - (16) becomes particularly transparent if $\tilde{\Gamma}$ is independent on the 3-momentum $\vec{P}$. Then, using $M^{2}=P^{2}-R e \Sigma^{r e t}$ as an independent variable instead of the energy $P_{0} \equiv \varepsilon$, Eq. (16) turns to

$$
\frac{d M_{i}^{2}}{d t}=\frac{M_{i}^{2}-M_{0}^{2}}{\tilde{\Gamma}_{(i)}} \frac{d \tilde{\Gamma}_{(i)}}{d t}
$$

for the time evolution of the test-particle $i$ in the invariant mass squared [64,65]. Now the deviation from the pole mass, i.e. $\Delta M^{2}=M^{2}-M_{0}^{2}$, follows the equation

$$
\frac{d}{d t} \Delta M^{2}=\frac{\Delta M^{2}}{\tilde{\Gamma}} \frac{d}{d t} \tilde{\Gamma} \leftrightarrow \frac{d}{d t}\left(\ln \left(\frac{\Delta M^{2}}{\tilde{\Gamma}}\right)\right)=0,
$$

which expresses the fact that the off-shellness in mass scales with the total width.

In order to demonstrate the importance of off-shell transport dynamics we present in Fig. 3 the time evolution of the mass distribution of $\rho$ (upper part) and $\omega$ (lower part) mesons for central $\mathrm{C}+\mathrm{C}$ collisions $(\mathrm{b}=1 \mathrm{fm})$ at $2 \mathrm{~A} \mathrm{GeV}$ for the dropping mass + collisional broadening scenario (as an example). The l.h.s. of Fig. 3 corresponds to the calculations with on-shell propagation whereas the r.h.s. show the results for the off-shell dynamics.

As seen from Fig. 3 the initial $\rho$ and $\omega$ mass distributions are quite broad even for a small system such as $C+C$ where, however, the baryon density at $2 \mathrm{~A} \mathrm{GeV}$ may reach (in some local cells) up to $2 \rho_{0}$. The number of vector mesons decreases with time due to their decays and the absorption by baryons $(\rho N \rightarrow \pi N$ or $\rho N \rightarrow \pi \pi N)$. Most of the $\rho$ mesons decay/disappear already inside the 'fireball' for density $\rho_{N}>0$. Due to the 'fireball' expansion the baryon density drops quite fast, so some amount of $\rho$ 

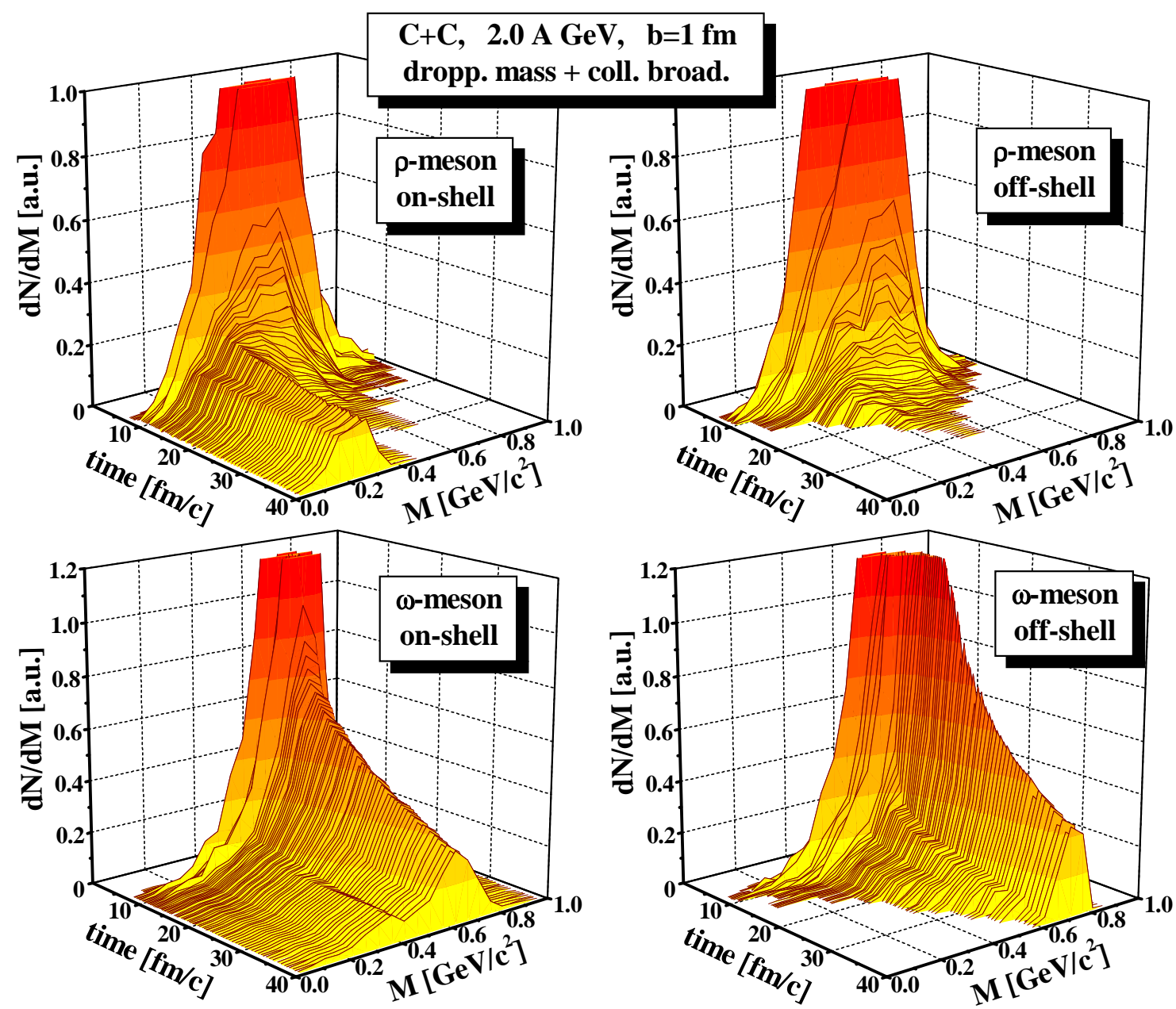

Fig. 3. Time evolution of the mass distribution of $\rho$ (upper part) and $\omega$ (lower part) mesons for central $C+C$ collisions ( $\mathrm{b}=1 \mathrm{fm}$ ) at $2 \mathrm{~A} \mathrm{GeV}$ for the dropping mass + collisional broadening scenario. The l.h.s. of Fig. 3 correspond to the calculations with on-shell dynamics whereas the r.h.s. show the off-shell HSD results.

mesons reach the very low density zone or even the 'vacuum'. Since for the off-shell case (r.h.s. of Fig. 3) the $\rho$ spectral function changes dynamically by propagation in the dense medium according to Eqs. (14)-(16), it regains the vacuum shape for $\rho \rightarrow 0$. This does not happen for the on-shell treatment (1.h.s. of Fig. 33); the $\rho$ spectral function does not change its shape by propagation but only by explicit collisions with other particles. Indeed, there is a number of $\rho$ 's which survive the decay or absorption and leave the 'fireball' with masses below $2 m_{\pi}$.

Accordingly, the on-shell treatment leads to the appearance of $\rho$ mesons in the vacuum with $M \leq 2 m_{\pi}$, which can not decay to two pions; thus they live practically 'forever' since the probability to decay to other channels is very small. Indeed, such $\rho$ 's will continuously shine low mass dileptons which leads to an unphysical 'enhancement/divergence' of the dilepton yield at low masses (note, that the dilepton yield is additionally enhanced by a factor $\left.\sim 1 / M^{3}\right)$. 
The same statements are valid for the $\omega$ mesons (cf. lower part of Fig. 33): since the $\omega$ is a long living resonance, a larger amount of $\omega$ 's survives with an in-medium like spectral function in the vacuum (in case of on-shell dynamics). Such $\omega$ 's with $M<$ $3 m_{\pi}$ can decay only to $\pi \gamma$ or electromagnetically (if $M<m_{\pi}$ ). Since such unphysical phenomena appear in on-shell transport descriptions (including an explicit vectormeson propagation) an off-shell treatment is mandatory.

The off-shell equations of motion (14) - (16) are the present standard for the HSD transport approach and have also been adopted by the Rossendorf group in Ref. [57].

\subsection{Vector meson production in the nuclear medium}

Apart from the explicit off-shell propagation the production of mesons is also modified in the nuclear medium in line with their in-medium spectral function. We mention that in-medium mass shifts of particles have been traditionally incorporated in on-shell transport approaches by parametrizing the vacuum cross sections as a function of the invariant energy $\sqrt{s}$ and the threshold $\sqrt{s_{0}}$ (cf. 20,2176]). In-medium modifications of cross sections then in first order have been incorporated by a shift of the 'pole' threshold (which corresponds to the pole mass of the spectral function) $\sqrt{s_{0}^{*}}=\sqrt{s_{0}}+M_{0}^{*}-M_{0}$ due to a lack of microscopic calculations for the corresponding in-medium transition matrix elements.

In order to account for the in-medium effects in production cross sections, we model the vector meson production cross sections in $N N$ and $\pi N$ reactions in the following way: The total cross section $\sigma_{N N \rightarrow V N N}\left(s, \rho_{N}\right)\left(\operatorname{similar} \sigma_{\pi N \rightarrow V N}\left(s, \rho_{N}\right)\right)$ is

$$
\sigma_{N N \rightarrow V N N}\left(s, \rho_{N}\right)=\int_{M_{\min }}^{M_{\max }} d M \frac{d \sigma_{N N \rightarrow V N N}\left(s, M, \rho_{N}\right)}{d M} .
$$

The mass differential cross sections are approximated by

$$
\frac{d \sigma_{N N \rightarrow V N N}\left(s, M, \rho_{N}\right)}{d M}=\sigma_{N N \rightarrow V N N}^{0}\left(s, M, \rho_{N}\right) \cdot A\left(M, \rho_{N}\right) \cdot \frac{\int_{M_{\min }}^{M_{\max }} A\left(M, \rho_{N}\right) d M}{\int_{M_{\text {min }}}^{M_{\text {lim }}} A\left(M, \rho_{N}\right) d M}
$$

where $A\left(M, \rho_{N}\right)$ denotes the meson spectral function (11) for given total width $\Gamma_{V}^{*}$ (6); $M_{\max }=\sqrt{s}-2 m_{N}$ is the maximal kinematically allowed invariant mass of the vector meson $V$ and $m_{N}$ is the mass of the nucleon. In Eq. (21) $\sigma_{N N \rightarrow V N N}^{0}\left(s, M, \rho_{N}\right)$ is the parametrization (based on phase space) of the vacuum cross section [21] with a modified 'pole' threshold $\sqrt{s_{0}^{*}}\left(\rho_{N}\right)=2 m_{N}+M_{0}^{*}\left(\rho_{N}\right)$ instead of $\sqrt{s_{0}}=2 m_{N}+M_{0}$ in the vacuum. Note, that the physical threshold for the $\rho$ production, e.g., in $N N$ 

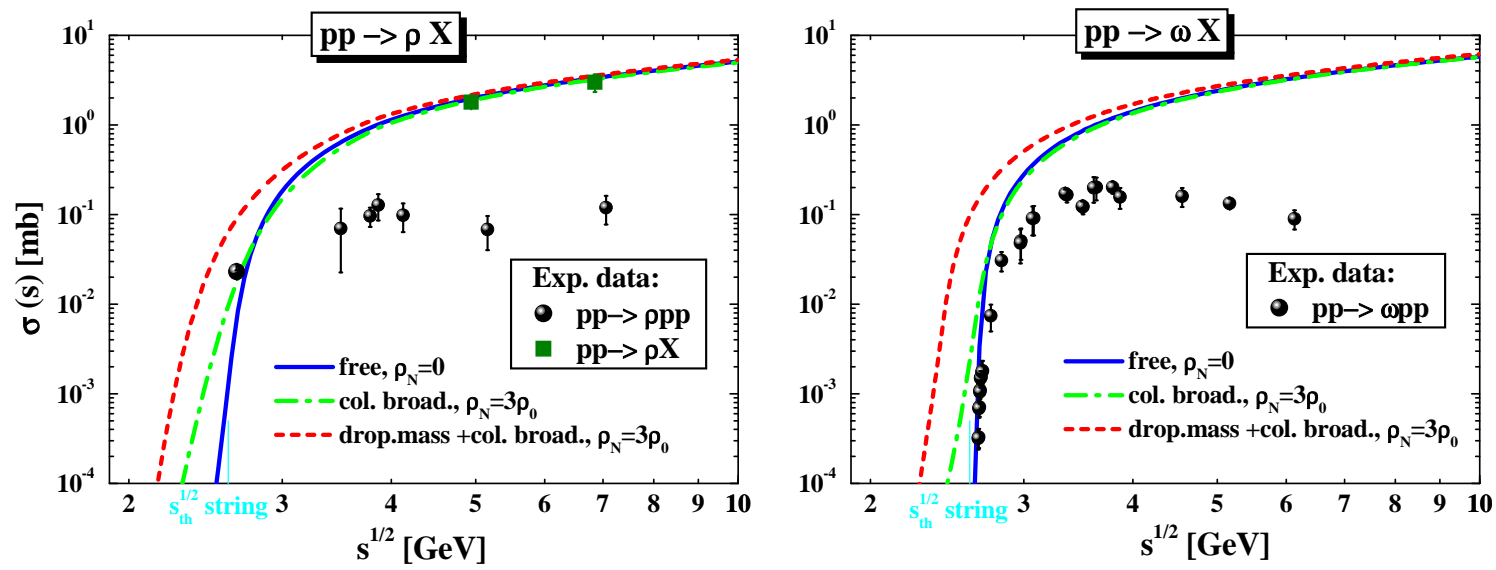

Fig. 4. The production cross sections for the channels $N N \rightarrow V X$ as a function of the invariant energy $\sqrt{s}$ for densities $\rho_{N}=0$ and $3 \rho_{0}$. The solid lines represent the parametrizations of inclusive $N N \rightarrow V X$ cross sections in free space while the dash-dotted lines stand for the 'collisional broadening' scenario; the dashed lines show the inclusive cross sections in the 'dropping mass + collisional broadening' scenario. The vertical thin blue line displays the threshold for meson production by string formation and decay $\left(\sqrt{s}_{t h}=2.6 \mathrm{GeV}\right)$ in case of baryon-baryon channels. The experimental data $77 / 78,88$ are shown for exclusive $p p \rightarrow V p p$ (dots) and inclusive $p p \rightarrow V X$ (squares) vector meson production where $X$ stands for two baryons and further mesons.
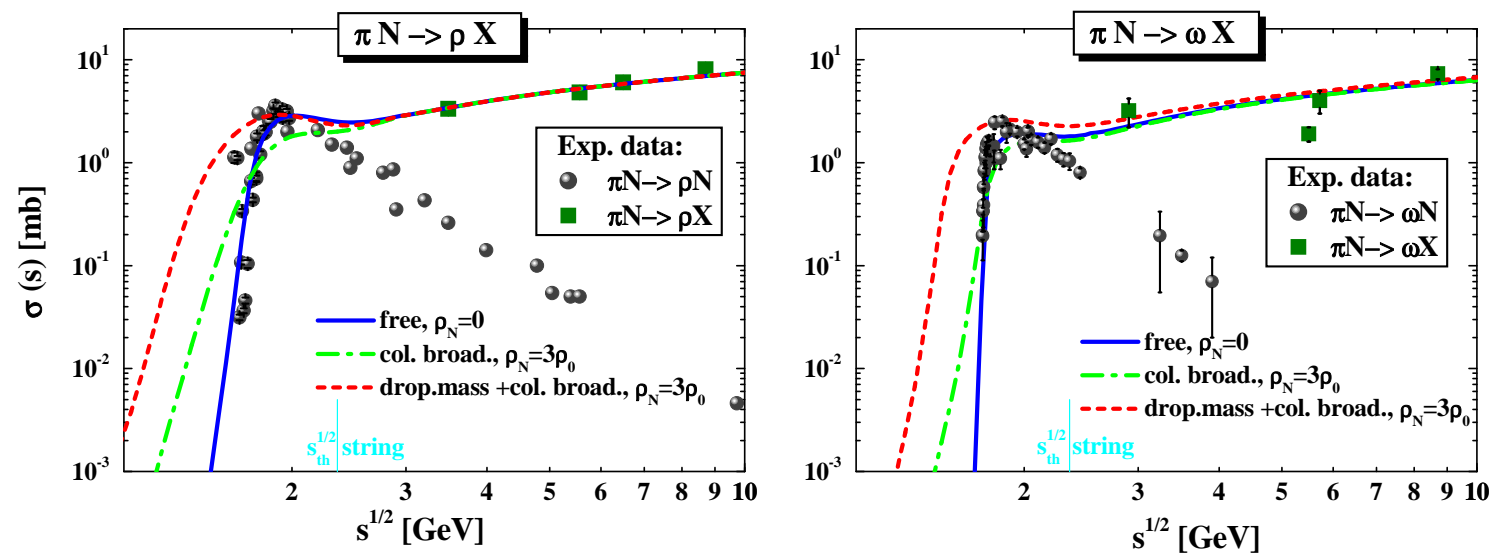

Fig. 5. The production cross sections for the channels $\pi N \rightarrow V N(V=\rho, \omega)$ as a function of the invariant energy $\sqrt{s}$ for densities $\rho_{N}=0$ and $3 \rho_{0}$. The solid lines represent the parametrizations of inclusive $\pi N \rightarrow V X$ cross sections in free space while the dash-dotted lines stand for the 'collisional broadening' scenario. The dashed lines show the inclusive cross sections in the 'dropping mass + collisional broadening' scenario. The experimental data [7788] are shown for exclusive $\pi N \rightarrow V N$ (dots) and inclusive $\pi N \rightarrow V X$ (squares) vector meson production. The vertical light blue line shows the threshold for meson production by string formation and decay $\left(\sqrt{s}_{t h}=2.3 \mathrm{GeV}\right)$ as implemented in HSD for meson-baryon channels.

reactions is defined as $\sqrt{s}_{t h}=2 m_{N}+M_{\text {min }}$, i.e. for the broadening scenario $\sqrt{s}_{t h} \rightarrow$ $2 m_{N}$. Thus, the formula (21) is used to model the vector meson production in $N N$ reactions in the vacuum and in the medium. 
The results for the channels $N N \rightarrow V X$ are displayed in Fig. [4 as a function of the invariant energy $\sqrt{s}$ for densities $\rho_{N}=0$ and $\rho_{N}=3 \rho_{0}$. The solid lines represent the parametrizations for inclusive $(N N \rightarrow V X)$ cross sections in free space while the dash-dotted lines stand for the 'collisional broadening' scenario; the dashed lines show the inclusive cross sections in the 'dropping mass + collisional broadening' scenario. The light vertical blue line stands for the threshold for meson production by string formation and decay $\left(\sqrt{s}_{t h}=2.6 \mathrm{GeV}\right)$ for baryon-baryon channels. The squares show the experimental data [77,78,88] for the inclusive $N N \rightarrow V X$ vector meson production (here $X$ stays for all final particles including two baryons and further mesons). The dots correspond to the experimental data for the exclusive $p p \rightarrow V p p$ reaction.

The string formation and decay reasonable matches the inclusive $(N N \rightarrow V X)$ data for $\rho$ (squares) taken from Refs. [77/78/88]. It is seen that the excitation function of the vector meson production cross section in the 'collisional broadening' scenario is basically smeared out close to threshold. Only when incorporating additionally a dropping mass the thresholds are shifted down in energy such that the production cross sections become enhanced in the subthreshold regime with increasing nuclear density $\rho_{N}$.

The actual results for the channels $\pi N \rightarrow V N(V=\rho, \omega)$ are displayed in Fig. 5 as a function of the invariant energy $\sqrt{s}$ for densities $\rho_{N}=0$ and $\rho_{N}=3 \rho_{0}$. Here the solid lines represent the parametrizations of $\pi N \rightarrow V X$ cross sections in free space while the dash-dotted lines stand for the 'collisional broadening' scenario. The dashed lines show the inclusive cross sections in the 'dropping mass + collisional broadening' scenario. The experimental data are displayed for exclusive $\pi N \rightarrow V N$ (dots) and inclusive $\pi N \rightarrow V X$ (squares) vector meson production channels. The vertical light blue line stands for the threshold for meson production by string formation and decay $\left(\sqrt{s}_{t h}=2.3 \mathrm{GeV}\right)$ as implemented in HSD for meson-baryon channels. It is seen that the string decay reasonable matches the inclusive $(\pi N \rightarrow V X)$ data for $\rho$ and $\omega$ (squares) taken from Refs. [77,88].

One observes from Fig. 5 that in the 'collisional broadening' scenario the excitation function of the vector meson production cross section is only smeared out close to threshold due to the broader vector meson width at finite density. As in case of $N N$ reactions the thresholds are shifted down in energy when incorporating additionally a dropping mass. Since the collisional broadening is low for the $\phi$ meson the modifications of the production cross section for $\phi$ 's stay very moderate (not shown explicitly).

A note of caution has to be added here because the present parametrizations for vector meson production in the nuclear medium are not controlled by microscopic studies on the various production channels for the case of finite nuclear density $\rho_{N}$. A consistent microscopic description of in-medium vector meson production cross sections thus is urgently needed. Furthermore, it should be noted that the experimental determination of a $\rho$ meson production cross section is problematic since this 
would imply to measure, e.g., the full invariant mass range of $\pi^{+} \pi^{-}$pairs in a $p$ wave final state. This is a rather complicated task because the two-pion decay width $\Gamma_{\pi \pi}$ approaches zero close to the two-pion threshold where the data are dominated by pions in a relative $s$-wave. Accordingly experimental data have to be considered simultaneously with the operational definition for a ' $\rho$-meson'.

We also mention that in the early study [45] we have considered the possible contribution from the Dalitz decay of the $N(1520)$ resonance: $N(1520) \rightarrow \rho N \rightarrow e^{+} e^{-} N$. At those times this process has been treated perturbatively, i.e. without explicit propagation of the $N(1520)$. We obtained from our qualitative estimates that the dilepton radiation from the $N(1520)$ in the hot and dense medium is strongly reduced due to a 'melting' of the $N(1520)$. Since the width and pole position of the $N(1520)$ is still quite uncertain we do not include the contribution of the $N(1520)$ explicitly and rather rely on the findings in [45] that the $N(1520)$ decay contribution to dileptons should become small in a dense medium.

\section{4 'In-medium' extension of the LUND string model}

The particle production from baryon-baryon collisions above $\sqrt{s}_{t h} \simeq 2.6 \mathrm{GeV}$ and from meson-baryon collisions above $\sqrt{s}_{t h} \simeq 2.3 \mathrm{GeV}$ are modeled in HSD via string formation and decay. The actual realization is the LUND string-fragmentation model FRITIOF [67] which is known to describe inelastic hadronic reactions in a wide energy regime at zero baryon density. In the standard version of FRITIOF the resonances are produced according to the vacuum Breit-Wigner spectral function (by default nonrelativistic) with a constant width. Moreover, the Breit-Wigner shape is truncated symmetrically around the pole mass, $\left|M-M_{0}\right|<\delta$, with $\delta$ chosen 'properly' for each particle such that no problems are encountered in the particle decay chains [67].

However, in order to study in-medium effects at energies close to the individual thresholds $\sqrt{s}_{t h}$ one has to incorporate the relativistic in-medium spectral functions (as described in Section 2.1 for the vector mesons) also consistently in the string fragmentation model. Such an extension of the FRITIOF model is performed in this study for the first time and the resonance production in the medium via FrITIOF is treated with the full relativistic Breit-Wigner spectral functions including the density dependent self-energy and in-medium width (depending on mass and baryon density). Also the truncation of the spectral function in mass is removed, i.e. the resonance mass is chosen within the physical thresholds from $M_{\min }$ to $M_{\max }$. As before the total energy and momentum conservation holds strictly in the extended Fritiof model.

We mention that this extension of the Lund model - which allows to implement any shapes of spectral functions into the fragmentation scheme - has been used not only for vector mesons but also for the $\Delta$ resonance in order to incorporate the mass-dependent width $\Gamma_{\Delta}\left(M_{\Delta}\right)$ (in line with the low energy part of HSD, where 
the parametrization from Ref. [46] has been used for $\left.\Gamma_{\Delta}\left(M_{\Delta}\right)\right)$, which leads to a hardening of the high mass dilepton spectra from the $\Delta$ Dalitz decay. Note, that 'inmedium' effects - i.e. collisional broadening or/and dropping masses - are accounted only for the vector mesons $(\rho, \omega, \phi)$ in the present study.

\subsection{Time integration (or 'shining') method for dilepton production}

Since dilepton production is a very rare process (e.g. the branching ratio for the vector meson decay is $\sim 10^{-5}$ ), a perturbative method is used in order to increase statistics. In the HSD approach (in this study as well as in our earlier investigations [21,44,45,48,49]) we use the time integration (or 'shining') method first introduced by $\mathrm{Li}$ and $\mathrm{Ko}$ [71]. The main idea of this method is that dileptons can be emitted during the full lifetime of the resonance $R$ before its strong decay into hadrons or absorption by the surrounding medium. For example, the $\rho^{0}$ decay (with invariant mass $M)$ to $e^{+} e^{-}$during the propagation through the medium from the production time $t=0$ up to the final ('death') time $t_{F}$ - which might correspond to an absorption by baryons or to reactions with other hadrons as well as the strong decay into two pions - is calculated as

$$
\frac{d N^{\rho \rightarrow e^{+} e^{-}}}{d M}=\sum_{t=0}^{t_{F}} \Gamma^{\rho^{0} \rightarrow e^{+} e^{-}}(M) \cdot \frac{\Delta t}{\gamma(\hbar c)} \cdot \frac{1}{\Delta M}
$$

in the mass bin $\Delta M$ and time step $\Delta t$ (in $\mathrm{fm} / \mathrm{c}$ ). In (22) $\gamma$ is the Lorentz factor of the $\rho$-meson with respect to the calculational frame. The electromagnetic decay width is defined as

$$
\Gamma^{\rho^{0} \rightarrow e^{+} e^{-}}(M)=C_{\rho} \frac{M_{0}^{* 4}}{M^{3}},
$$

where $C_{\rho}=\Gamma^{\rho \rightarrow e^{+} e^{-}}\left(M_{0}\right) / M_{0}$. Here $M_{0}$ is the vacuum pole mass, $M_{0}^{*}$ is the inmedium pole mass which is equal to the vacuum pole mass for the collisional broadening scenario, however, is shifted for the dropping mass scenario according to Eq. (10).

The time integration method allows to account for the full in-medium dynamics of vector mesons from production ('birth') up to their 'death'. We note that by calculating the dilepton emission only at the strong decay vertex (e.g. as in Refs. [50,51]) the dilepton rate (as well as the density dependence of the dilepton emission) is underestimated since a sizeable part of the emission history (e.g. before the absorption point by baryons or before the decay to pions) is lost. 


\section{6 $e^{+} e^{-}$bremsstrahlung in elementary reactions}

The implementation of $e^{+} e^{-}$bremsstrahlung from hadronic reactions in transport approaches has been limited so far to the dominant $p n$ channel in the 'soft photon' approximation (with phase-space corrections) [21,22,40,41,42] or even been neglected completely [50,55]. As we will demonstrate below this contribution should be reexamined and revised accordingly.

The soft-photon approximation (SPA) [79] is based on the assumption that the radiation from internal lines is negligible and the strong interaction vertex is on-shell. In this case the strong interaction part and the electromagnetic part can be separated, however, the cross section for dileptons has to be corrected [79] by reducing the phase-space for the colliding particles in their final-state. The phase-space corrected soft-photon cross section for the reaction $1+2 \rightarrow 1+2+e^{+}+e^{-}$can be written as

$$
\begin{aligned}
& \frac{d \sigma}{d y d^{2} q_{T} d M}=\frac{\alpha^{2}}{6 \pi^{2}} \frac{\bar{\sigma}(s)}{M q_{0}^{2}} \frac{R_{2}\left(s_{2}\right)}{R_{2}(s)}, \\
& R_{2}(s)=\sqrt{1-\left(m_{1}+m_{2}\right)^{2} / s} \\
& s_{2}=s+M^{2}-2 q_{0} \sqrt{s} \\
& \bar{\sigma}(s)=\frac{s-\left(m_{1}+m_{2}\right)^{2}}{2 m_{1}^{2}} \sigma(s),
\end{aligned}
$$

where $m_{1}$ is the mass of the charged accelerated particle, $M$ is the dilepton invariant mass, $q_{0}$ the energy, $q_{T}$ the transverse momentum and $y$ the rapidity of the dilepton pair. In (24) $\sigma(s)$ is the on-shell elastic cross section for the reaction $1+2 \rightarrow 1+2$.

In spite of the general limitation of the SPA - discussed in detail in Refs. [79,80] - Eq. (24) has been widely used for the calculation of the bremsstrahlung dilepton spectra by different transport groups $[21,22,40,41,42]$. Note, at those early times the applicability of the SPA for an estimate of dilepton radiation from $N N$ collisions at 1-2 GeV bombarding energies has been supported by independent One-BosonExchange (OBE) model calculations by Schäfer et al. [81] and later on by Shyam et al. [82] (cf. Fig. 6). In these models the effective parameters have been adjusted to describe elastic $N N$ scattering at intermediate energies. The models have then been applied to bremsstrahlung processes including the interference of different diagrams for the dilepton emission from all charged hadrons. As shown in Ref. [81] the $p p$ bremsstrahlung is much smaller than $p n$ bremsstrahlung due to a destructive interference of amplitudes from the initial and final radiation. We note that 'gauge invariant' results have been obtained in Refs. [81,82] by 'gauging' the phenomenological form factors at the meson-baryon vertices. However, there are different schemes to introduce 'gauge invariance' in OBE models - as stressed by Kondratyuk and Scholten [84] - which lead to sizeably different cross sections.

One has to point out that already in 1997 an independent study by de Jong et al. [83] 
- based on a full T-matrix approach - has indicated that the validity of the SPA for $e^{+} e^{-}$bremsstrahlung at intermediate energies of 1-2 GeV may be very questionable. However, in Ref. [83] only the $p p$ reaction has been considered (cf. r.h.s. of Fig. 6); indeed, the bremsstrahlung in the full T-matrix approach is larger by a factor of about 3 than the corresponding SPA calculations (and OBE results).
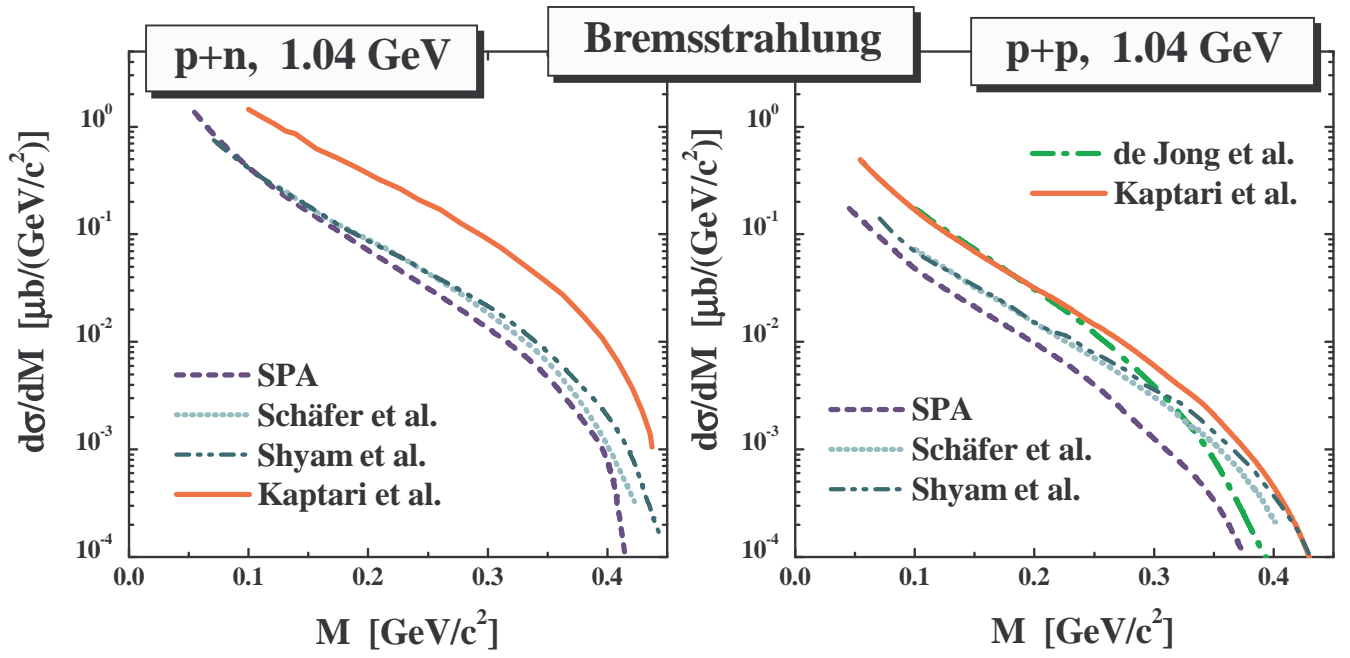

Fig. 6. The $e^{+} e^{-}$bremstrahlung from $p n$ (l.h.s.) and $p p$ (r.h.s.) channels. The dashed lines show the SPA results [79, the dotted and dashed-dot-dotted lines correspond to the OBE calculations by Schäfer et al. [81] and Shyam et al. 82, respectively. The dash-dotted line (r.h.s.) displays the $T$-matrix result from de Jong et al. 83] while the red solid lines show the calculations by Kaptari et al. 69].

Recently, new covariant OBE calculations for dilepton bremsstrahlung have been performed by Kaptari and Kämpfer [69]. The effective parameters for $N N$ scattering have been taken similar to [82], however, the restoration of gauge invariance has been realized in a different way. As mentioned above, there are several prescriptions for restoring gauge invariance in effective theories including momentum-dependent form factors in interactions with charged hadrons [84] and the actual results very strongly depend on the prescription employed. The scheme in Ref. [69] is to include explicitly the vertex form factors into the Ward-Takahashi identity for the full meson-exchange propagators, which is different from the method used in Refs. [81,82.

The $p n$ and $p p$ bremsstrahlung differential spectra from Kaptari et al. [69] are shown in Fig. 6 by the solid red lines. One can see that the difference between the Kaptari model and the SPA and OBE [81,82] results is up to a factor of 4 for $p n$ reactions and about a factor of 3 for $p p$ collisions at $1 \mathrm{GeV}$. Note, that the $p p$ bremsstrahlung from [69] is on the same level as the $T$-matrix calculations from Ref. [83] (cf. Fig. 6. r.h.s.). Thus, according to the Kaptari model the contributions from $p n$ and $p p$ bremsstrahlung have been substantially underestimated in the previous transport model calculations [21,22] (accounting only for elastic scattering events with charged hadrons).

We stress that formula (24) is only applicable for the bremsstrahlung radiation from 
elementary elastic scattering reactions (i.e. elastic $N N$ or $\pi N$ collisions). However, in the transport models of Refs. [56,57] it has been adopted also for inelastic $N N$ reactions which faces the problem of double counting since the dilepton emission from inelastic $N N$ reactions is dominantly taken into account by decays of the produced particles (i.e. $\Delta, \eta, \omega$ Dalitz decays, direct $\rho, \omega, \phi$ decay etc.). On the other hand, electromagnetic radiation also emerges from inelastic channels since each charged particle (that is decelerated or accelerated in a collision) will radiate dileptons. Due to an interference of all amplitudes there is a partial cancellation between initial state amplitudes and final state amplitudes in case of equal charges due to a different sign in the acceleration. On the other hand two hadrons with opposite charges in the final state will also interfere destructively such that general estimates for a wide class of inelastic hadronic reactions cannot easily be performed. In this respect the strategy in Refs. 5657] (to account for radiation from inelastic channels) is a very crude assumption whereas our approximation implies to disregard such additional radiation amplitudes in case of dominant electromagnetic decays which are taken into account explicitly. Due to a lack of microscopic calculations on the $T$-matrix level for radiation from channels with further charged particles in the final state we have to wait for respective experimental results to verify/falsify the different approximations.

In the present study we will adopt the results from Ref. [69] for the elementary bremsstrahlung processes. As will be shown in the next Sections this will have a sizeable impact on our results for the dilepton radiation from $A A$ collisions at $1-2$ $\mathrm{A} \cdot \mathrm{GeV}$.

We have to point out, furthermore, that in order to separate the bremsstrahlung $\left(p p \rightarrow p p e^{+} e^{-}\right)$from a vector-dominance like dilepton production via the $\rho$-meson $\left(p p \rightarrow p p \rho, \rho \rightarrow e^{+} e^{-}\right)$, we do not employ a vector-dominance formfactor when calculating the bremsstrahlung. Thus, the dilepton radiation via the decay of the virtual photon $\left(p p \rightarrow p p \gamma^{*}, \gamma^{*} \rightarrow e^{+} e^{-}\right)$and the direct $\rho$ decay to $e^{+} e^{-}$are distinguished explicitly in the calculations. This is different from the calculations of the Tübingen group [47] which have no dynamical $\rho$ meson but only a medium-dependent formfactor in the bremsstrahlung channel.

In closing this Subsection we stress that new calculations of dilepton bremsstrahlung - based on a consistent full $G$ - (or $T$-) matrix approach - are urgently needed in order to clarify the unsatisfactory situation. From the experimental side high statistics measurements for multi-differential dilepton spectra from elementary $p p$ and $p n(p d)$ reactions are required to settle this open question for elastic as well as inelastic channels. 


\section{$3 \quad \pi^{0}$ and $\eta$ production}

Since the low mass dilepton spectra are dominated by the Dalitz decays of $\pi^{0}$ and $\eta$ an explicit control of their production cross section is mandatory for a proper interpretation of dilepton mass spectra. We recall that the early suggestion of the DLS Collaboration that the enhanced $\mathrm{e}^{+} e^{-}$production seen at the BEVELAC in $C+C$ and $C a+C a$ collisions might be due to an enhanced production of $\eta$ 's was rejected by the TAPS Collaboration measuring the $\eta$ cross section via the $\pi^{0} \gamma$ decay channel for the same systems [85]. The TAPS argument has been confirmed by the transport calculations in Refs. [44,86].

In the last decade the experimental information of $\eta$ production in $p p$ and $p n$ reactions - especially close to threshold - has been drastically improved such that more appropriate parametrizations of these cross sections have to be implemented in transport in order to stay in line with the experimental progress. The presently available experimental information from exclusive $p p$ and $p n$ reactions is displayed in Fig. 7 by the full symbols as a function of the invariant energy above threshold and indicates that the $p n$ cross section is larger than the $p p$ cross section for $\eta$ production by up to a factor of 6 . Only at sufficiently high energy the initial isospin no longer plays any role due to multiple associated pion production. The solid and dashed lines in Fig. 7 show the novel parametrizations/results from HSD for $p p$ and $p n$ reactions which smoothly join the inclusive $p N \rightarrow \eta X$ production from string decay at high energy and are compatible with the low energy exclusive $p N \rightarrow \eta p N$ data from Refs. [87,88,89].

The number of $\pi^{0}$ 's and $\eta$ 's from HSD for ${ }^{12} C+{ }^{12} C$ at $1 \mathrm{~A} \cdot \mathrm{GeV}$ and $2 \mathrm{~A} \cdot \mathrm{GeV}$ are shown in Figs. 8 and 9 as a function of the impact parameter $b$ (lower blue lines). The average number of $\pi$ 's and $\eta$ 's is given by,

$$
<N_{x}>\left.\right|_{b_{\max }}=\frac{2 \pi \int_{0}^{b_{\max }} N_{x}(b) b d b}{\pi b_{\max }^{2}}
$$

where $b_{\max }$ denotes the maximum impact parameter considered in the calculation and $x=\left(\pi^{0}, \eta\right)$. The corresponding results for these quantities are displayed in Figs. 8 and 9 by the red squares as a function of $b=b_{\max }$ and demonstrate that the average number of $\pi^{0}$ 's and $\eta$ 's from the TAPS Collaboration in inclusive ${ }^{12} C+{ }^{12} C$ reactions (shaded areas) are reasonably reproduced by the transport calculations. Note that the inclusive reaction cross section is given by $\sigma_{\text {incl }}=\pi b_{\max }^{2}$.

In line with Figs. 8 and 9 we show in Fig. 10 the average $\pi^{0}$ and $\eta$ multiplicities for ${ }^{12} C+{ }^{12} C$ from 0.8 to $2 \mathrm{~A} \cdot \mathrm{GeV}$ from the TAPS Collaboration [91] (full squares) in comparison to the corresponding HSD results (stars). Since the agreement is acceptable (within error bars) we may proceed with the actual dilepton studies. 


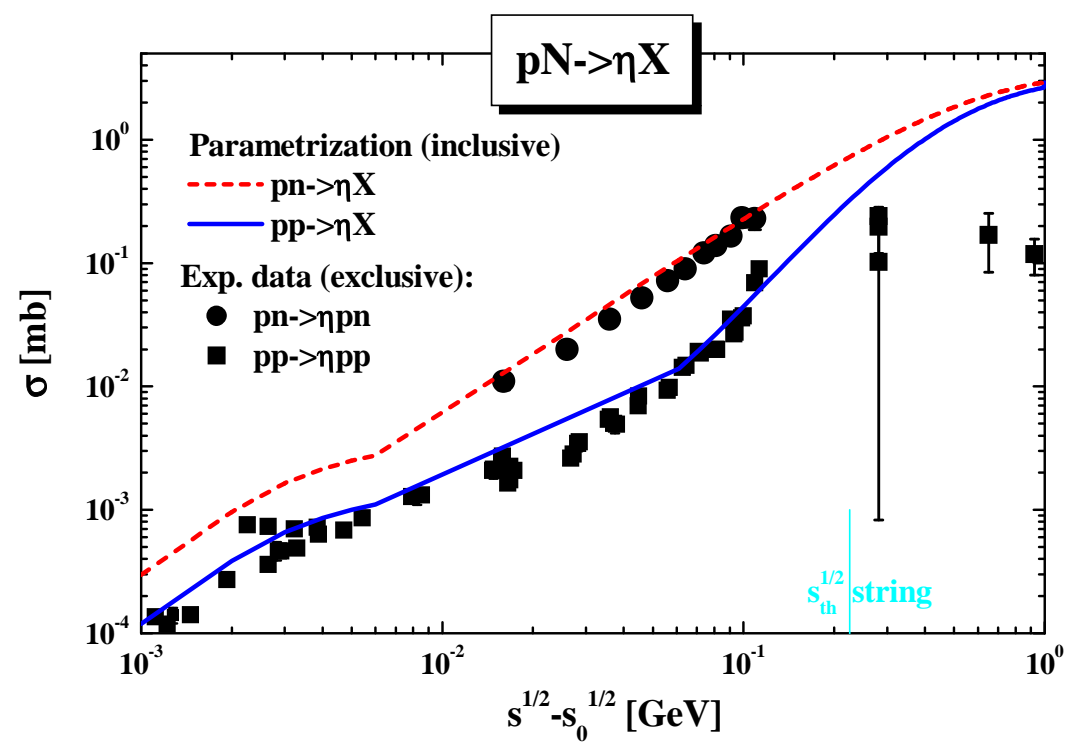

Fig. 7. The $\eta$ production cross section from $p p$ and $p n$ reactions as a function of the invariant energy above threshold. The solid and dashed lines represent the corresponding inclusive $(p N \rightarrow \eta X)$ parametrizations/results from the HSD transport approach, whereas the full symbols show the exclusive $(p N \rightarrow \eta p N)$ experimental data from Refs. 8788 89].
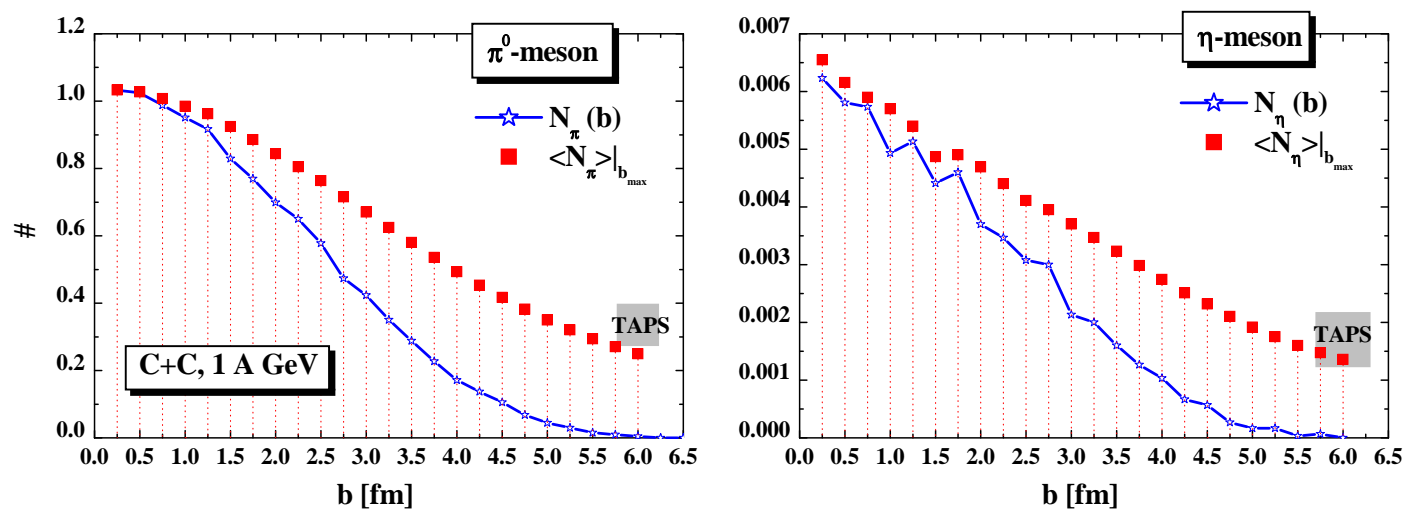

Fig. 8. The number of $\pi^{0}$ 's (l.h.s.) and $\eta$ 's (r.h.s.) from HSD for ${ }^{12} C+{ }^{12} C$ at $1 \mathrm{~A} \cdot \mathrm{GeV}$ as a function of the impact parameter $b$ (lower blue lines). The red squares denote the average number of $\pi$ 's and $\eta$ 's according to Eq. (25) for $b_{\max }=b$ and show that the average number of $\pi^{0}$ 's and $\eta$ 's from the TAPS Collaboration in inclusive ${ }^{12} C+{ }^{12} C$ reactions (shaded areas) is approximately reproduced for $b_{\max } \approx 6 \mathrm{fm}$.

\section{Dilepton production in $p p$ and $p d$ reactions}

Before coming to explicit results for nucleus-nucleus collisions we consider dilepton production in more elementary reactions like $p p$ and $p d$ in the energy range of interest. The results for $e^{+} e^{-}$production in $p p$ reactions are shown in Fig. 11 at bombarding energies of $1.04,1.27,1.61,1.85,2.09$ and $4.88 \mathrm{GeV}$ in comparison to the data from the BEVALAC [92] including the DLS acceptance filter and mass resolution [92. 

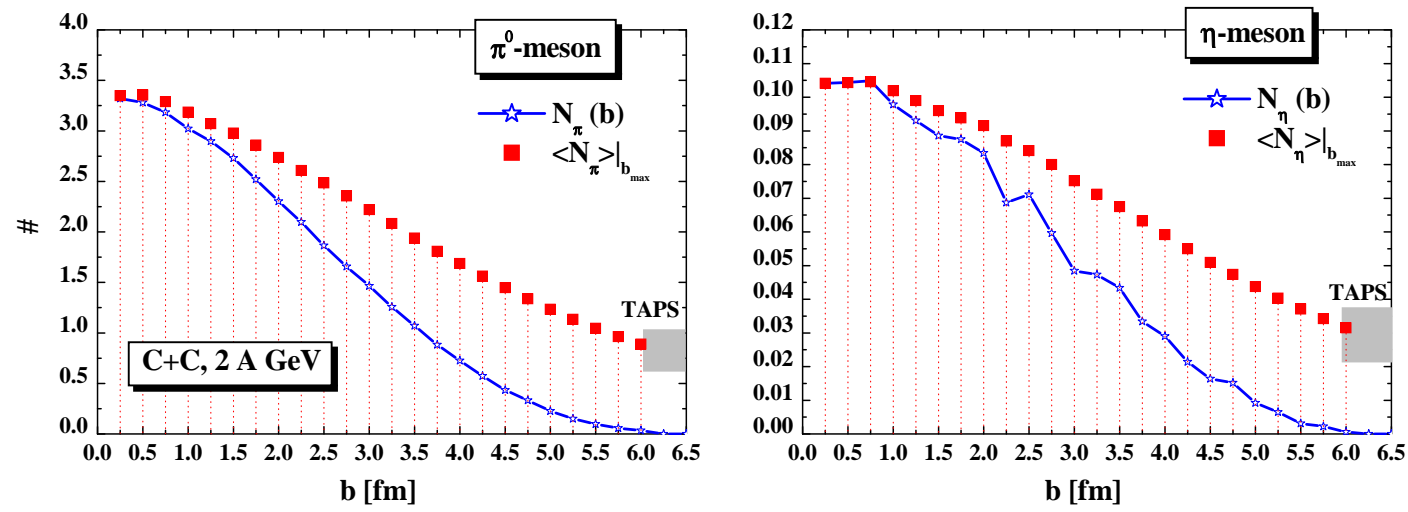

Fig. 9. The same as Fig. 8 for ${ }^{12} C+{ }^{12} C$ reactions at $2 \mathrm{~A} \cdot \mathrm{GeV}$.

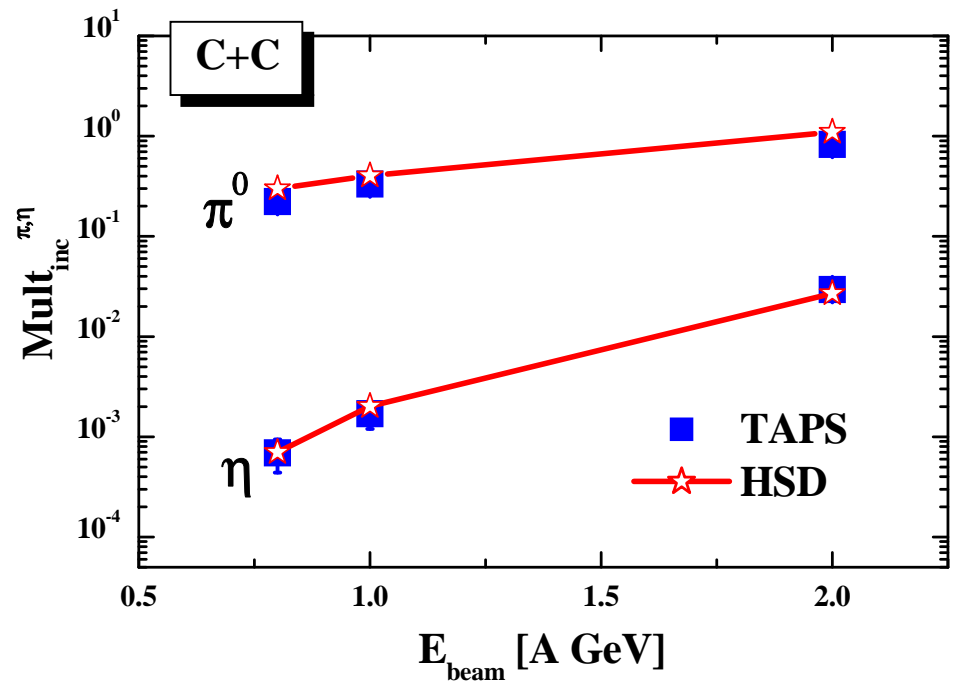

Fig. 10. The average $\pi^{0}$ and $\eta$ multiplicities for ${ }^{12} C+{ }^{12} C$ from 0.8 to $2 \mathrm{~A} \cdot \mathrm{GeV}$ from the TAPS Collaboration [91. (full squares) in comparison to the corresponding HSD results (stars).

The various contributions from the different channels (in the HSD calculations) are indicated additionally (see color coding in the figure). At all energies the $\pi^{0}$ Dalitz decay dominates for invariant masses up to the pion mass whereas the contributions from 0.2 to $0.6 \mathrm{GeV}$ vary drastically with energy. At 1.09 and $1.27 \mathrm{GeV}$ here the $\Delta$ Dalitz decay dominates which is superseeded by the $\eta$ Dalitz decay already at $1.61 \mathrm{GeV}$. The $p p$ bremsstrahlung contribution also gives a sizeable yield to the total dilepton cross section at the lower energies. At $2.09 \mathrm{GeV}$ we find that the calculations overestimate the yield from the $\rho$ and $\omega$ decays which has to be kept in mind when comparing to nucleus-nucleus data at $2 \mathrm{~A} \cdot \mathrm{GeV}$ (see below). At $4.88 \mathrm{GeV}$ all channels result from string excitation and decay (except bremsstrahlung) which is sufficiently well in line with the BEVALAC data. We note that at all energies the contribution from the direct Dalitz decay of heavy resonances $R \rightarrow e^{+} e^{-} N$ is suppressed compare to $\Delta \rightarrow e^{+} e^{-} N$ as pointed out before in Ref. [94]. 

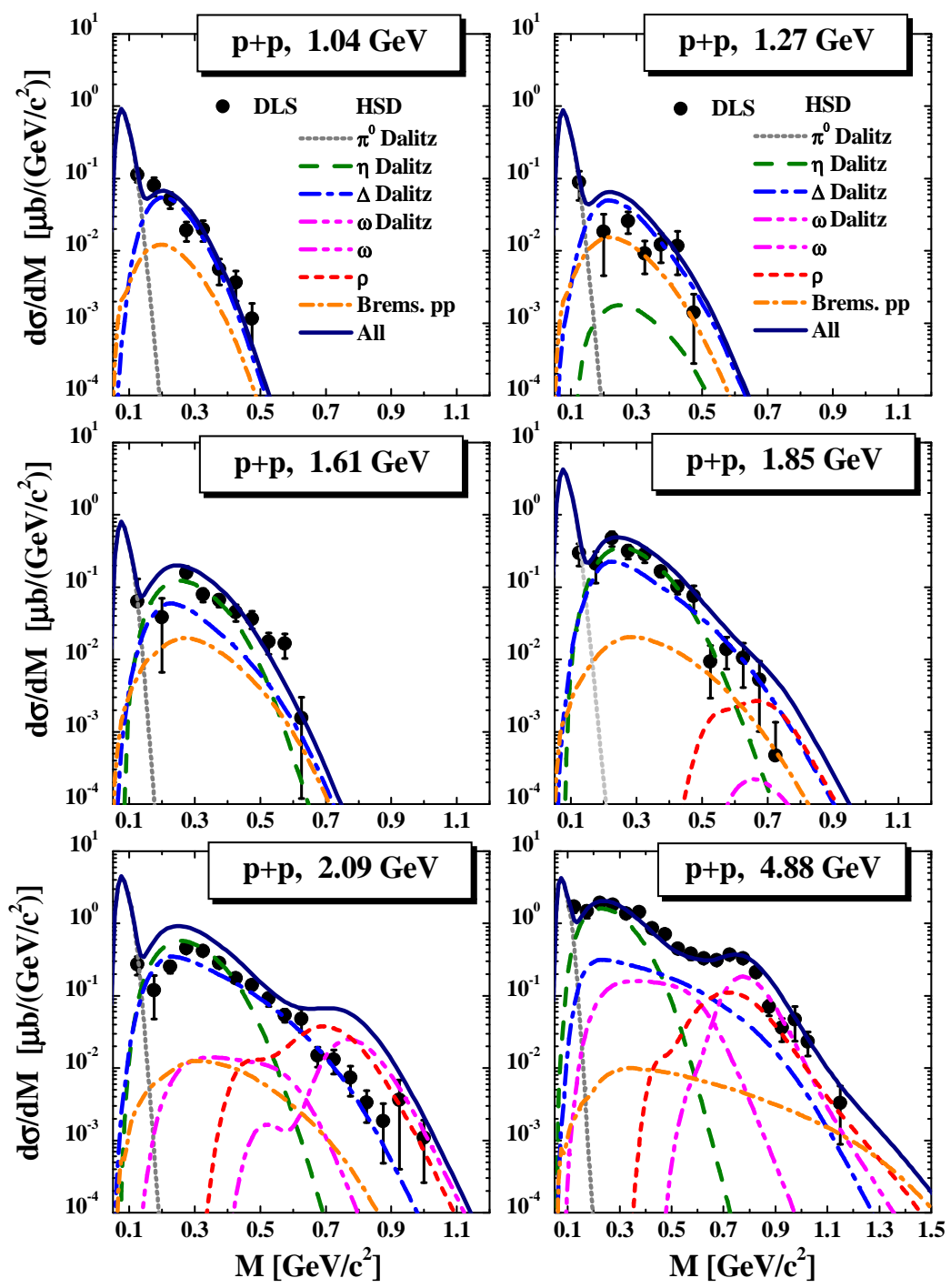

Fig. 11. The differential cross section for $e^{+} e^{-}$production in $p p$ reactions at bombarding energies of $1.04,1.27,1.61,1.85,2.09$ and $4.88 \mathrm{GeV}$ in comparison to the data from the BEVALAC [92] including the DLS acceptance filter and mass resolution [92]. The various contributions from the different channels (in the HSD calculations) are indicated additionally (see color coding in the legend).

The mass-differential dilepton cross section for $p d$ reactions is compared in Fig. 12 to the BEVALAC data at the same bombarding energies including the DLS acceptance filter and mass resolution [92]. In this case the relative contribution from the various Dalitz decays and vector mesons decays stays about the same as for $p p$ reactions except the contribution from bremsstrahlung which at the lowest energies is of the same order as the $\Delta$ Dalitz decay. Note that the $\rho$ and $\omega$ production is overestimated at $2.09 \mathrm{GeV}$ as in the case of $p p$ reactions. Furthermore, coherent production of $\eta, \rho$ and $\omega$ on the deuteron has been discarded in the HSD calculations since the latter channels show up only very close to the individual production thresholds and become negligible in $A+A$ reactions. 

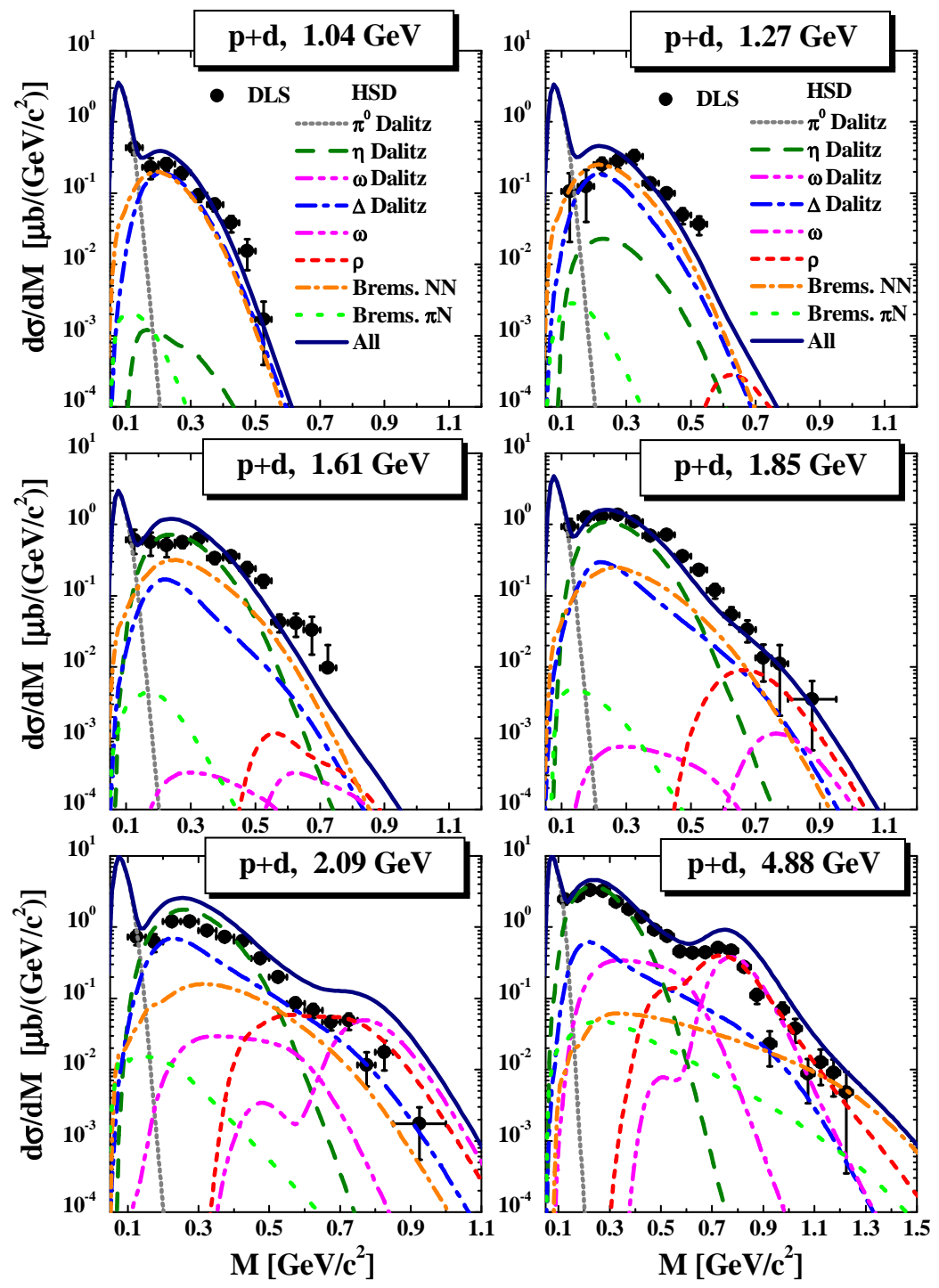

Fig. 12. The differential cross section for $e^{+} e^{-}$production in $p d$ reactions at bombarding energies of 1.04, 1.27, 1.61, 1.85, 2.09 and $4.88 \mathrm{GeV}$ in comparison to the data from the BEVALAC [92] including the DLS acceptance filter and mass resolution [92]. The various contributions from the different channels (in the HSD calculations) are indicated additionally (see color coding in the legend).

In view of the very limited DLS acceptance and mass resolution the overall description of these 'elementary' reactions is acceptable such that more complex reactions can be addressed. 


\section{Dilepton production in nucleus-nucleus collisions}

A major aim of the HADES Collaboration was to clarify the 'DLS-puzzle', i.e. to verify/falsify the DLS data [39] from the experimental side. Accordingly, the same systems have been reinvestigated at $1 \mathrm{~A} \cdot \mathrm{GeV}$ with the HADES detector in order to clarify the issue. The situation, however, is not as easy since the DLS and HADES acceptances differ significantly [39,61]. However, recently the HADES Collaboration has performed a direct comparison with the DLS data for $\mathrm{C}+\mathrm{C}$ at $1 \mathrm{~A} \mathrm{GeV}$ [39] by filtering the HADES data with the DLS acceptance [63]. Both measurements were found to agree very well [63]! Thus, the 'DLS puzzle' has been solved from the experimental side; there is no obvious contradiction between the DLS and HADES data! The major goal for the transport models now is to verify the solution of the 'DLS-puzzle' from the theoretical side. Note that transport calculations allow for a direct comparison between the DLS and HADES measurements by employing the different FILTER routines to the same set of calculated events.

\subsection{Comparison to the DLS data}

We start with a reinvestigation of the DLS data employing the novel cross sections for $\eta$ production, the modified $\Delta$ Dalitz decay contribution as well as the additional (and enhanced) bremsstrahlung channels as described above. The results of our transport calculation are displayed in Fig. 13 for ${ }^{12} C+{ }^{12} C$ at $1.04 \mathrm{~A} \cdot \mathrm{GeV}$ in case of 'free' vector-meson spectral functions (upper part) and in case of the 'dropping mass + collisional broadening' scenario (lower part) employing the DLS acceptance filter and mass resolution.

In fact, the situation has substantially improved compared to the early studies and the missing yield in the 'free' scenario is now reduced to a factor of about 1.5 in the mass region from 0.25 to $0.5 \mathrm{GeV}$. This is due to slightly higher contributions from $\Delta$ and $\eta$ Dalitz decays and a significantly larger yield from bremsstrahlung channels.

To see the effects of these modifications quantitatively, we show in Table 1 a comparison of our 'new' results with the 'old' ones from Refs. [44,45] - indicated as 'new'/'old' - for $\eta$ and $\Delta$ Dalitz decays, $N N$ Bremsstrahlung channels as well as for the sum of all contributions for $\mathrm{C}+\mathrm{C}$ at $1 \mathrm{~A} \mathrm{GeV}$ for invariant masses of $0.2,0.35$ and $0.5 \mathrm{GeV}$. Both results correspond to the 'free' scenario.

As seen from Fig. 13, the bremsstrahlung yield now is similar in shape and magnitude as the $\Delta$ Dalitz decay contribution and enhanced by factor up to 5 due to the novel $N N$ bremsstrahlung cross section from [69] and accounting for the contribution from $p p$ bremsstrahlung additionally to $p n$. The contribution of the pion-nucleon bremsstrahlung is quite small in the $\mathrm{C}+\mathrm{C}$ system due to the limited energy available in meson-baryon collisions and the moderate rescattering rate of pions. 

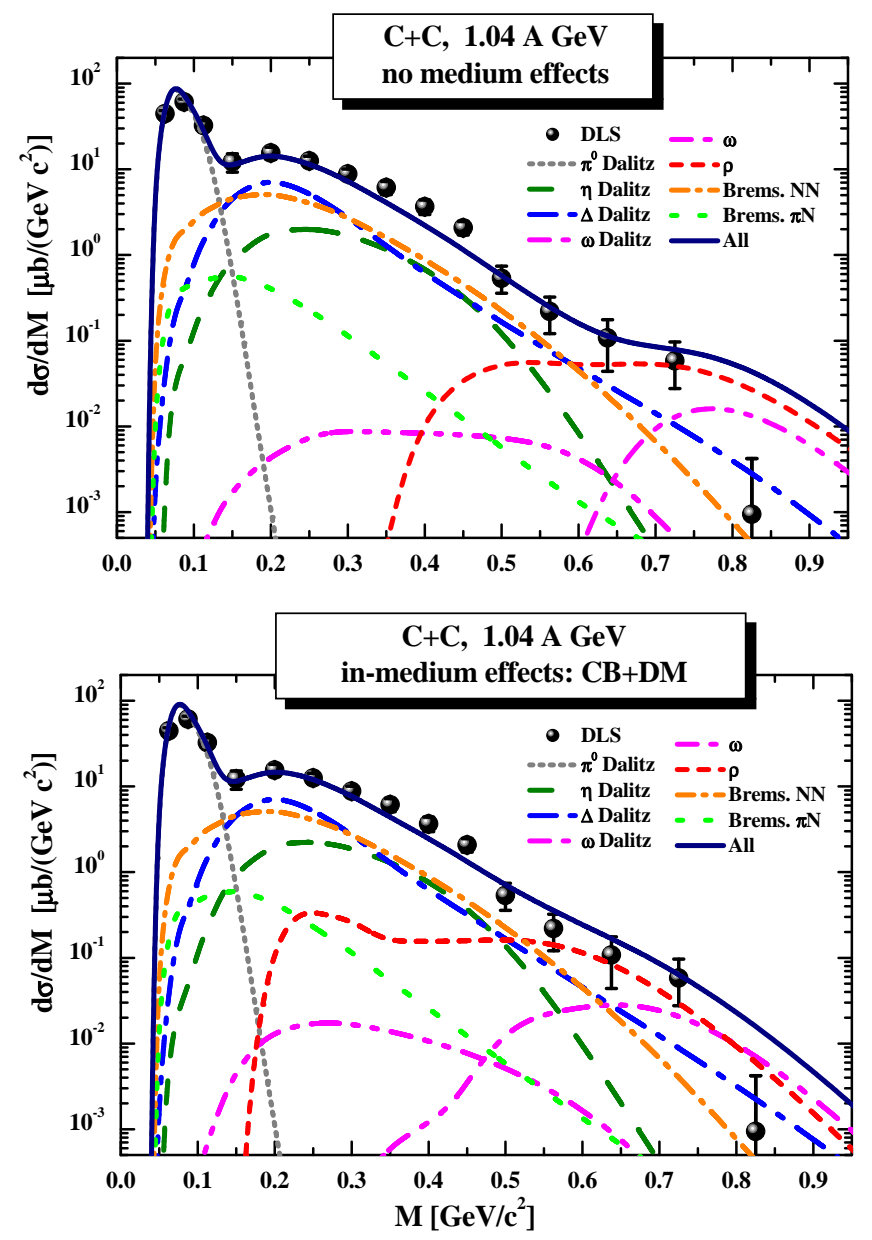

Fig. 13. Results of the HSD transport calculation for the mass differential dilepton spectra in case of ${ }^{12} C+{ }^{12} C$ at $1.04 \mathrm{~A} \cdot \mathrm{GeV}$ in comparison to the DLS data [39]. The upper part shows the case of 'free' vector-meson spectral functions while the lower part gives the result for the 'dropping mass + collisional broadening' scenario. In both scenarios the DLS acceptance filter and mass resolution have been incorporated. The different color lines display individual channels in the transport calculation (see legend).

As in our previous studies the $\eta$ Dalitz decay is subdominant. We obtain only a slight enhancement (up to $30 \%$ ) of the $\eta$ yield at $1 \mathrm{~A} \mathrm{GeV}$ compared to the early study [44] since modifications of the $\eta$ production cross section in $N N$ reactions (cf. Section 3) are relevant at higher energies. The enhancement of the high mass $\Delta$ Dalitz decay yield is related mainly to the modifications in the LUND string model by implementation of the mass dependent $\Delta$ width in the string fragmentation (cf. Section 2.4) which enhances the population of high mass $\Delta$ resonances. Additionally, we use now the parametrization from Ernst et al. [22] for the $\Delta \rightarrow N e^{+} e^{-}$decay.

Medium modifications for the vector mesons turn out to yield an enhancement in the region of $0.4<M<0.5 \mathrm{GeV}$ but are very moderate due to the light system $C+C$. Though a slightly better description of the DLS data is achieved it would be premature to claim the presence of in-medium effects on the vector mesons from these data. 
Table 1

The mass differential dilepton yield $\frac{d \sigma}{d M}[\mu \mathrm{b} / \mathrm{GeV}]$ for $\eta$ and $\Delta$ Dalitz decays, $N N$ bremsstrahlung as well as the sum of all contributions for $C+C$ at $1 \mathrm{~A} \mathrm{GeV}$. The left numbers in each column stand for the present results whereas the right numbers show the early results from Refs. 4445] - 'new'/'old'. The last column presents the DLS experimental data [39]. Both calculations refer to the 'free' scenario.

\begin{tabular}{|c|c|c|c|c|c|}
\hline $\mathrm{M}[\mathrm{GeV}]$ & $\eta$ & $\Delta$ & Brems. NN & sum & exp. data \\
\hline 0.2 & $1.75 / 1.3$ & $7.05 / 5.5$ & $5.0 / 1.88$ & $14.2 / 4.7$ & $15.5 \pm 2.6$ \\
0.35 & $1.15 / 0.9$ & $1.25 / 0.4$ & $1.6 / 0.35$ & $4.1 / 1.57$ & $6.1 \pm 1.1$ \\
0.5 & $0.12 / 0.11$ & $0.16 / 0.03$ & $0.22 / 0.04$ & $0.54 / 0.28$ & $0.54 \pm 0.2$ \\
\hline
\end{tabular}

Additional information is provided by the ${ }^{40} \mathrm{Ca}+{ }^{40} \mathrm{Ca}$ system at $1.04 \mathrm{~A} \cdot \mathrm{GeV}$ where the measured DLS spectra are reproduced in a qualitatively and quantitatively similar manner by the transport calculations (cf. Fig. 14) as in case of the lighter system. Again the combined bremsstrahlung channels provide a contribution in the same order as the $\Delta$ Dalitz decay; the $\eta$ Dalitz decay remains subdominant but the $\pi N$ bremsstrahlung increases compared to the $C+C$ system due to more frequent pion rescattering on nucleons. In-medium effects for the vector mesons can be indentified in the transport calculations but are hard to see in the total dilepton spectra.

Is the 'DLS puzzle' solved? This question can only be answered in a convincing manner by comparison with the recent HADES data.

\subsection{Comparison to HADES data}

The same multi-differential dilepton spectra - used for comparison with the DLS data - are now filtered by the HADES acceptance routines and smeared with the HADES mass resolution [61. A comparison of our calculations for the ${ }^{12} \mathrm{C}+{ }^{12} \mathrm{C}$ system at $1.0 \mathrm{~A} \cdot \mathrm{GeV}$ with the HADES data from Ref. [63] is presented in Fig. [15] and demonstrates that the agreement between data and transport calculations is reasonable for the HADES data, too. In this case the differential dilepton spectrum is divided by the average number of $\pi^{0}$ 's which in experiment is determined by half of the average number of $\left(\pi^{+}+\pi^{-}\right)$. The spectra look slightly different in shape due to the much higher acceptance at low invariant mass where the $\pi^{0}$ Dalitz decay practically exhausts the dilepton spectrum. As in case of the DLS data the $\eta$ Dalitz decay turns out to be subdominant and the $\Delta$ Dalitz decay to be of similar magnitude as the combined bremsstrahlung contribution. Again the effect of in-medium vectormeson spectral functions is hard to see in the total spectra.

A comparison to the HADES mass-differential dilepton spectra for ${ }^{12} C+{ }^{12} C$ at 2 $\mathrm{A} \cdot \mathrm{GeV}[61]$ is presented in Fig. 16 for the 'free' (upper part) and the in-medium 

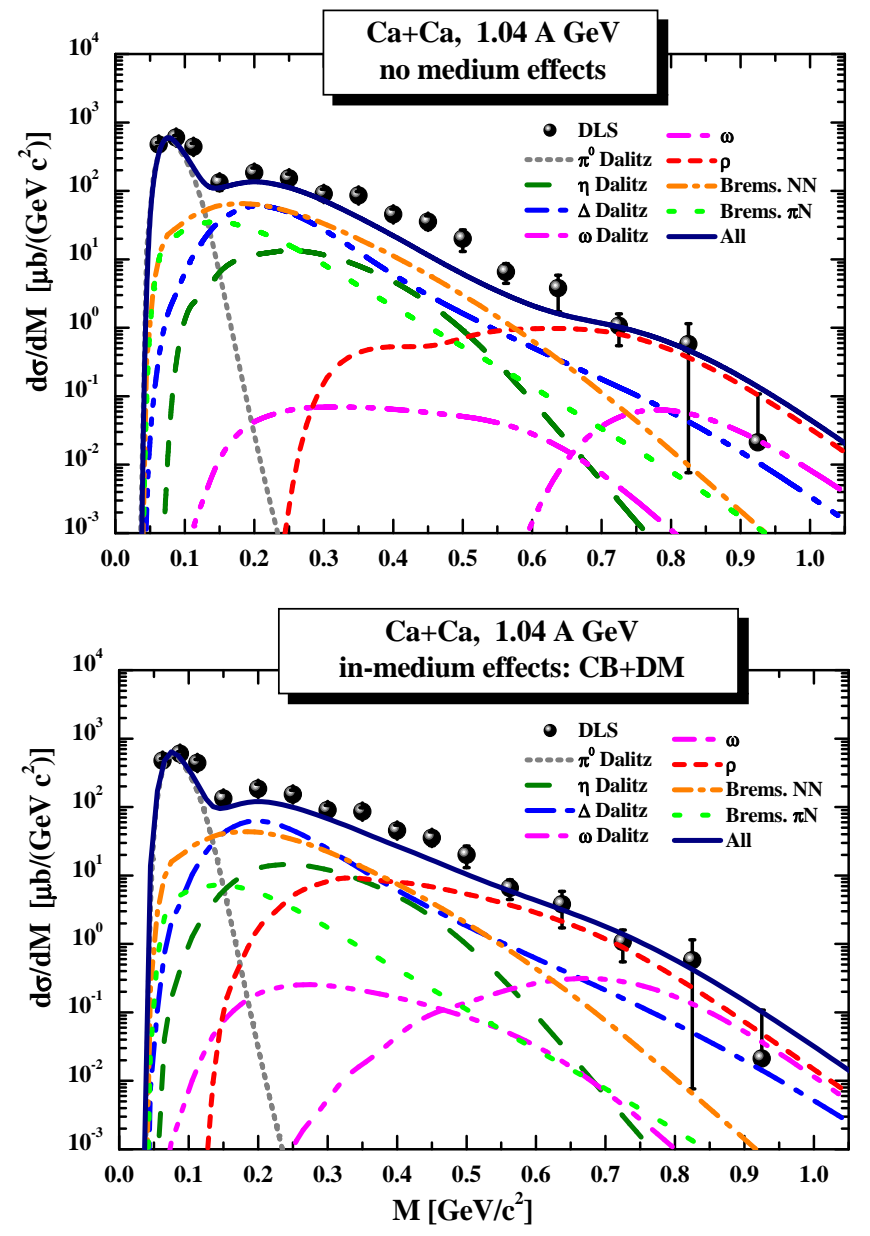

Fig. 14. The mass differential dilepton spectra from HSD in case of ${ }^{40} \mathrm{Ca}+{ }^{40} \mathrm{Ca}$ at 1.04 A. GeV in comparison to the DLS data [39]. The upper part shows the case of 'free' vector-meson spectral functions while the lower part gives the result for the 'dropping mass + collisional broadening' scenario. In both scenarios the DLS acceptance filter and mass resolution have been incorporated. The different color lines display individual channels in the transport calculation (see legend).

scenario (lower part). At the higher bombarding energy the $\eta$ Dalitz decay provides the dominant contribution in the mass region from 0.2 to $0.5 \mathrm{GeV}$ followed by $\Delta$ Dalitz decays and the combined bremsstrahlung channels. The mass region around $0.75 \mathrm{GeV}$ is overestimated in the 'free' scenario, whereas including in-medium spectral functions for the vector mesons the description of the data is improved (very similar to Ref. [57]) due a shifting of strength from the vector-meson mass regime to lower invariant mass. However, the in-medium effects for the light $C+C$ system are only very moderate.

We now turn back to the system ${ }^{12} C+{ }^{12} C$ at $1.04 \mathrm{~A} \cdot \mathrm{GeV}$ and ask the question if the agreement in Fig. 15 might be accidental? To address this issue we investigate the dilepton rapidity spectra in the center-of-mass system for different cuts on the invariant mass. The results of our transport calculations are shown in Fig. 17 where the mass integrated rapidity distributions are displayed on the upper panel, a cut 

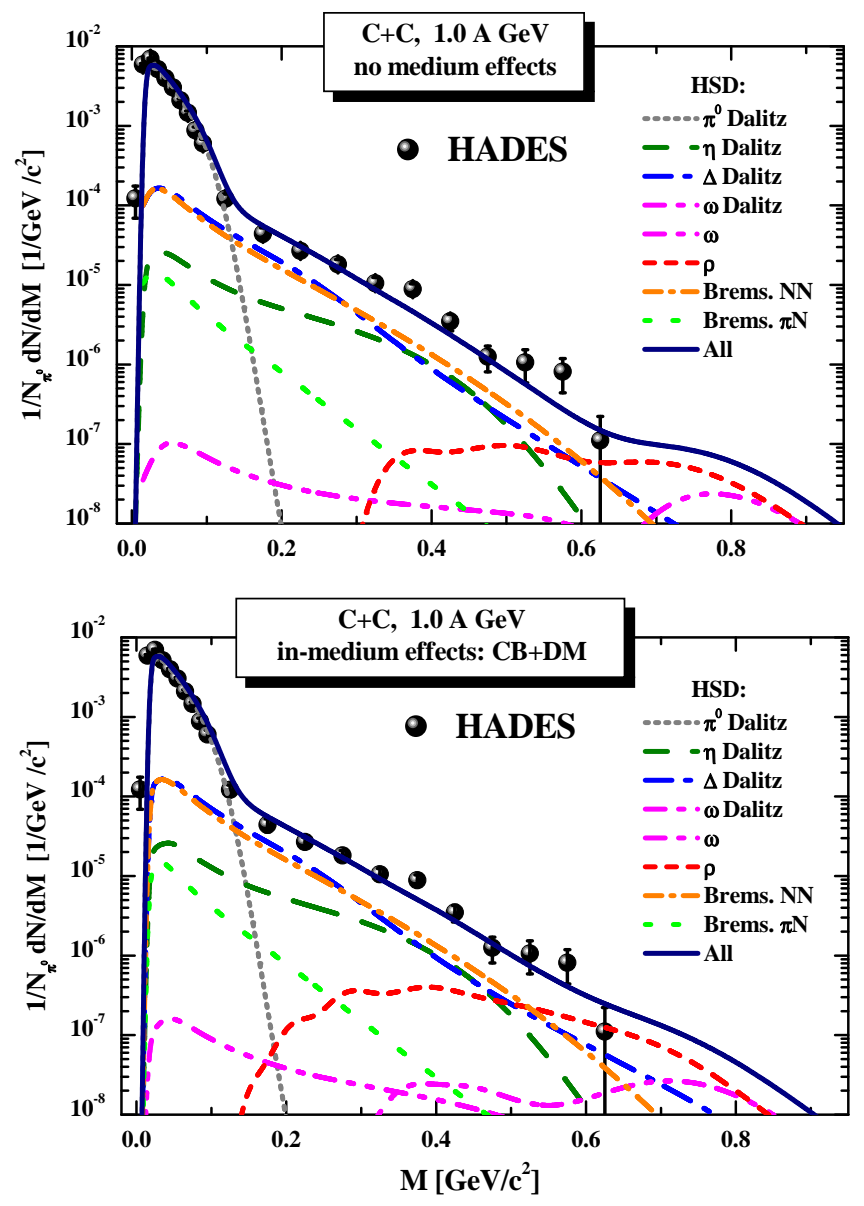

Fig. 15. Results of the HSD transport calculation for the mass differential dilepton spectra - divided by the average number of $\pi^{0}$ 's - in case of ${ }^{12} C+{ }^{12} C$ at $1.0 \mathrm{~A} \cdot \mathrm{GeV}$ in comparison to the HADES data [63]. The upper part shows the case of 'free' vector-meson spectral functions while the lower part gives the result for the 'dropping mass + collisional broadening' scenario. In both scenarios the HADES acceptance filter and mass resolution have been incorporated. The different color lines display individual channels in the transport calculation (see legend).

on low mass dileptons $(M<0.15 \mathrm{GeV})$ is performed in the middle part while the lower part shows the rapidity distribution for a cut $M>0.15 \mathrm{GeV}$. The individual colored lines display the contributions from the various channels in the HSD calculations. Since the experimental analysis for the dilepton rapidity spectra (involving the same cuts) is still in progress we still have to wait for the data to verify/falsify our predictions.

We recall that very similar investigations have been performed for ${ }^{12} C+{ }^{12} C$ at 2.0 A. GeV by the HADES Collaboration [62. Figure 18 shows their mass integrated rapidity distributions for dileptons from ${ }^{12} C+{ }^{12} C$ at $2.0 \mathrm{~A} \cdot \mathrm{GeV}$ (top panel) in comparison to the HSD calculations for the in-medium scenario. A cut on low mass dileptons $(M<0.15 \mathrm{GeV})$ is performed in the upper part, the middle panel corresponds to the cut $0.15 \mathrm{GeV}<M<0.55 \mathrm{GeV}$ while the lower part shows the dilepton 

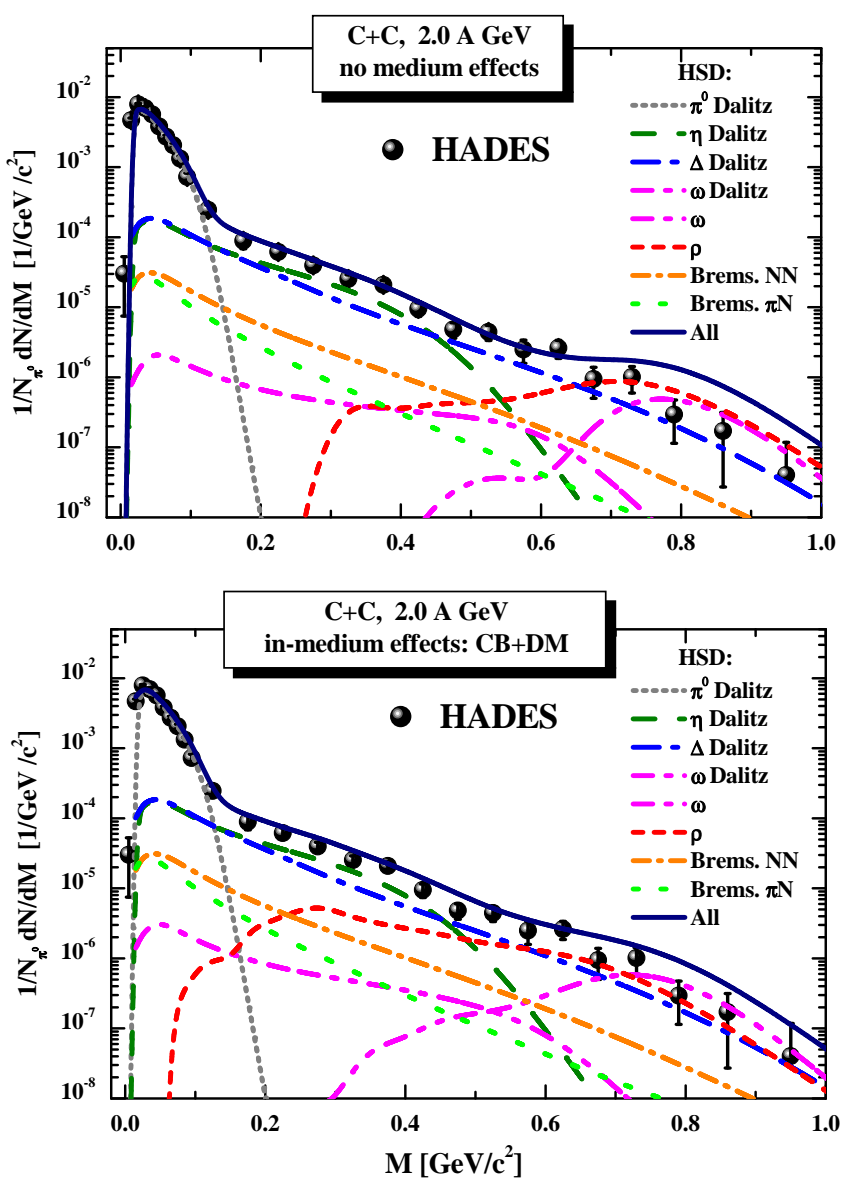

Fig. 16. The mass differential dilepton spectra from HSD in case of ${ }^{12} C+{ }^{12} C$ at $2 \mathrm{~A} \cdot \mathrm{GeV}$ in comparison to the HADES data [61]. The upper part shows the case of 'free' vector-meson spectral functions while the lower part gives the result for the 'dropping mass + collisional broadening' scenario. In both scenarios the HADES acceptance filter and mass resolution have been incorporated. The different color lines display individual channels in the transport calculation (see legend).

rapidity distribution for $M>0.55 \mathrm{GeV}$. As mentioned above, the $\eta$ Dalitz decay contribution becomes dominant in the interval $0.15<M<0.55 \mathrm{GeV}$ at $2.0 \mathrm{~A} \cdot \mathrm{GeV}$, whereas the contribution of bremsstrahlung decreases. In total we find the HSD calculations to provide a good description of the experimental rapidity distributions for all invariant mass ranges considered.

Apart from dilepton rapidity distributions (for different mass bins) also transverse momentum spectra of $e^{+} e^{-}$pairs provide additional information. For comparison with future HADES data we show the results of our HSD calculations in Fig. 19] for the dilepton transverse momentum spectra in the mass range $M<0.15 \mathrm{GeV}$ (upper part), $0.15 \leq M \leq 0.55 \mathrm{GeV}$ (middle part) and $M>0.55 \mathrm{GeV}$ (lower part) for ${ }^{12} \mathrm{C}+{ }^{12} \mathrm{C}$ at $1.0 \mathrm{~A} \cdot \mathrm{GeV}$. For the lowest invariant mass bin the transverse momentum spectrum is entirely dominated by the $\pi^{0}$ Dalitz decays, however, followed by the bremsstrahlung contribution at low $p_{T}$. In the interesting mass range 0.15 

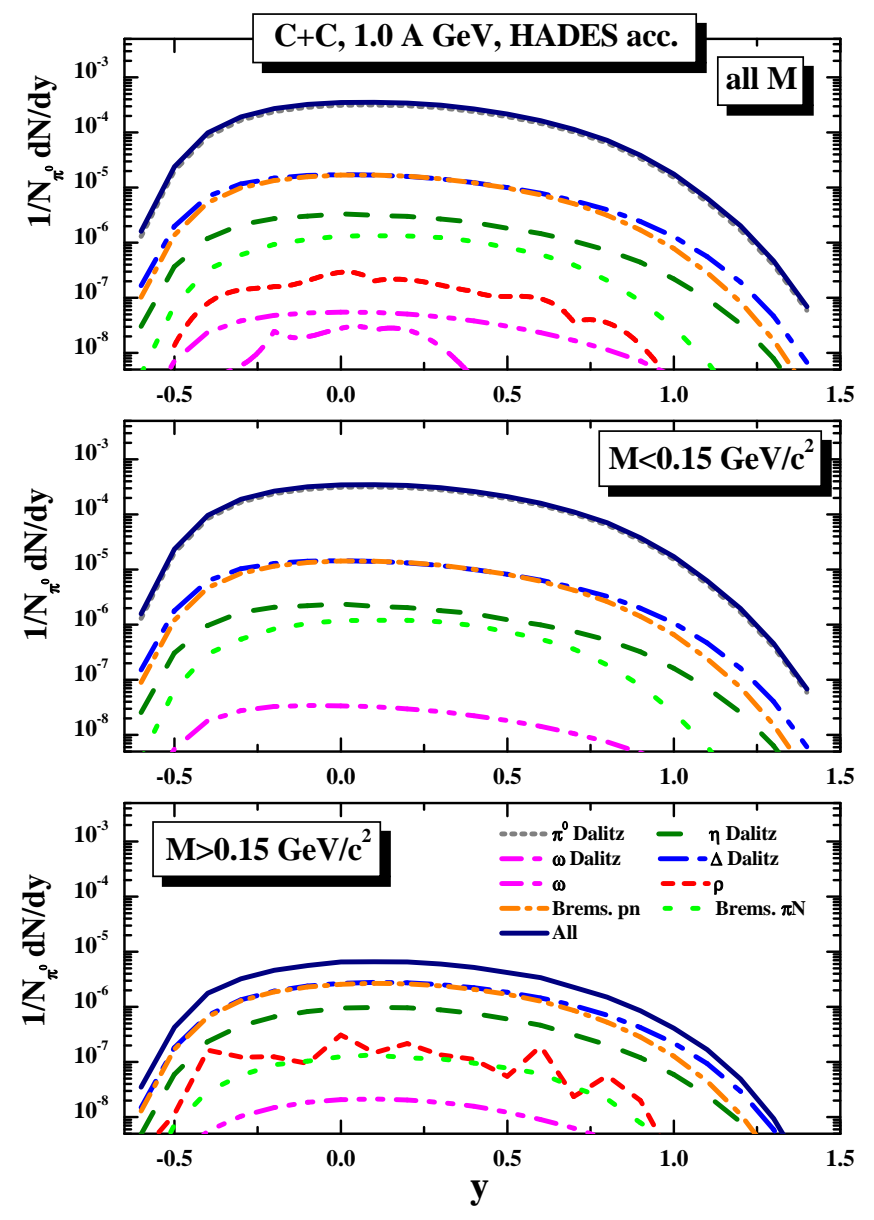

Fig. 17. HSD predictions for the mass integrated rapidity distributions for dileptons for ${ }^{12} C+{ }^{12} C$ at $1.0 \mathrm{~A} \cdot \mathrm{GeV}$ (top panel). A cut on low mass dileptons $(M<0.15 \mathrm{GeV})$ is performed in the middle part while the lower part shows the dilepton rapidity distribution for a cut $M>0.15 \mathrm{GeV}$. The individual colored lines display the contributions from the various channels in the HSD calculations (see color coding in the legend).

$<M<0.55 \mathrm{GeV}$ the bremsstrahlung and $\Delta$ Dalitz contributions dominate which might provide a handle to establish the strength of the bremsstrahlung channels independently from the mass differential spectra.

Whereas the data analysis for the dilepton $p_{T}$ spectra for ${ }^{12} C+{ }^{12} C$ at $1.04 \mathrm{~A} \cdot \mathrm{GeV}$ is still in progress we may gain related information in comparison to HADES data at 2.0 A. GeV. Accordingly, the dilepton transverse momentum spectra from the in-medium HSD calculations for ${ }^{12} C+{ }^{12} C$ at $2.0 \mathrm{~A} \cdot \mathrm{GeV}$ are shown in Fig. 20 in comparison to the data from the HADES Collaboration [62]. The upper part corresponds to the mass range $M \leq 0.15 \mathrm{GeV} / \mathrm{c}^{2}$, the middle part to $0.15 \leq M \leq 0.55 \mathrm{GeV} / \mathrm{c}^{2}$ and the lower part to $M \geq 0.55 \mathrm{GeV} / \mathrm{c}^{2}$. Indeed, we find again a quite reasonable agreement with the HADES data on the transverse momentum distributions for practically all mass intervals. A slight overestimation of the experimental data at lower $p_{T}$ is seen for the mass interval $M \geq 0.55 \mathrm{GeV} / \mathrm{c}^{2}$ (lower part of Fig. 201). This is due to the 

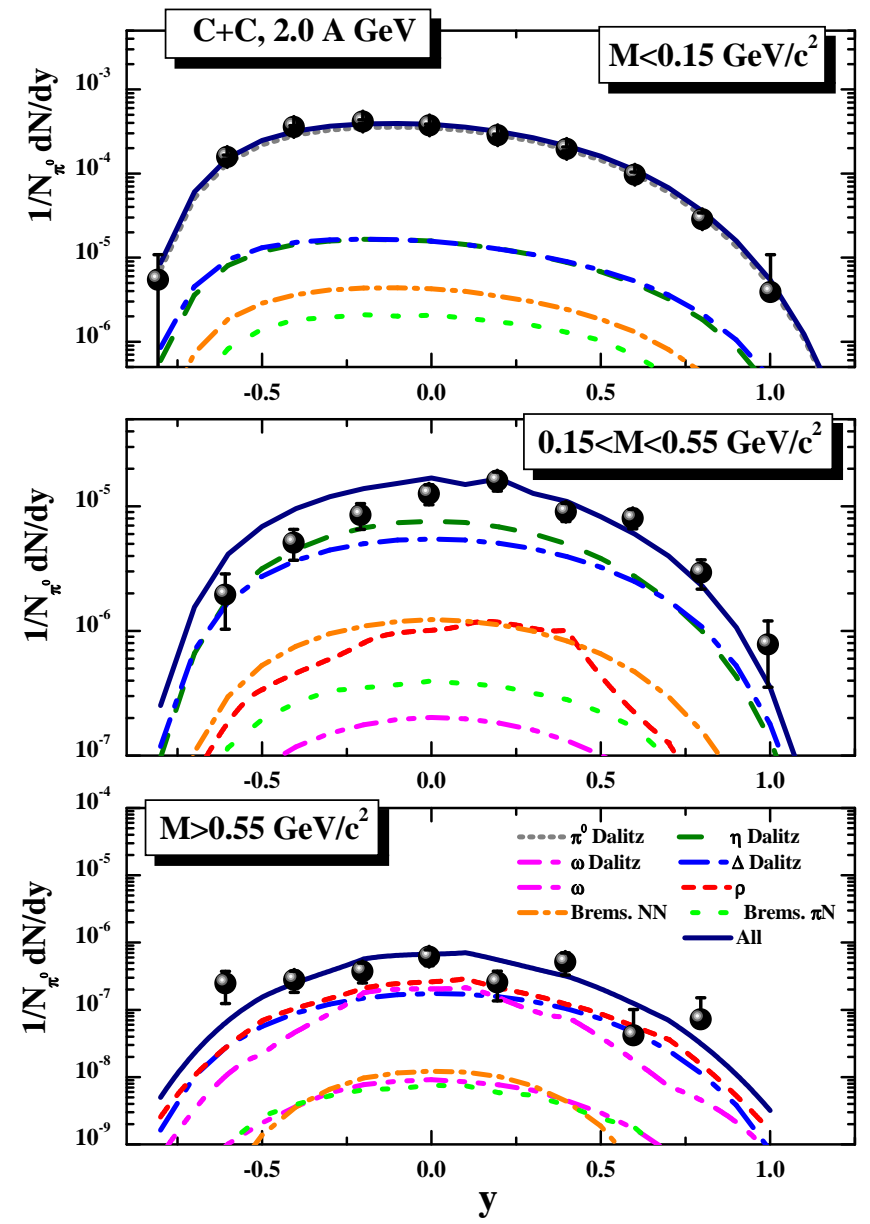

Fig. 18. The mass integrated rapidity distributions for dileptons from HSD for ${ }^{12} C+{ }^{12} C$ at $2.0 \mathrm{~A} \cdot \mathrm{GeV}$ (top panel) in comparison to the HADES data 62]. A cut on low mass dileptons $(M<0.15 \mathrm{GeV})$ is performed in the upper part, the middle panel corresponds to the cut $0.15 \mathrm{GeV}<M<0.55 \mathrm{GeV}$ while the lower part shows the dilepton rapidity distribution for $M>0.55 \mathrm{GeV}$. The individual colored lines display the contributions from the various channels in the HSD calculations (see color coding in the legend).

fact that the 'dropping mass + collisional broadening' scenario for the vector mesons leads to a shift of the $p_{T}$ spectra to the low $p_{T}$ region. This effect is stronger for heavy systems with larger baryon densities and might be used to distinguish the different in-medium scenarios from the experimental side.

\subsection{Predictions at SIS energies}

The DLS data on $e^{+} e^{-}$production from elementary reactions like $p p$ and $p d$ collisions have been taken in a rather small range of phase space with limited statistics. This is sufficient for 'pilot experiments' but decisive measurements - allowing for a distinction between different model scenarios - are still missing. To this end the 

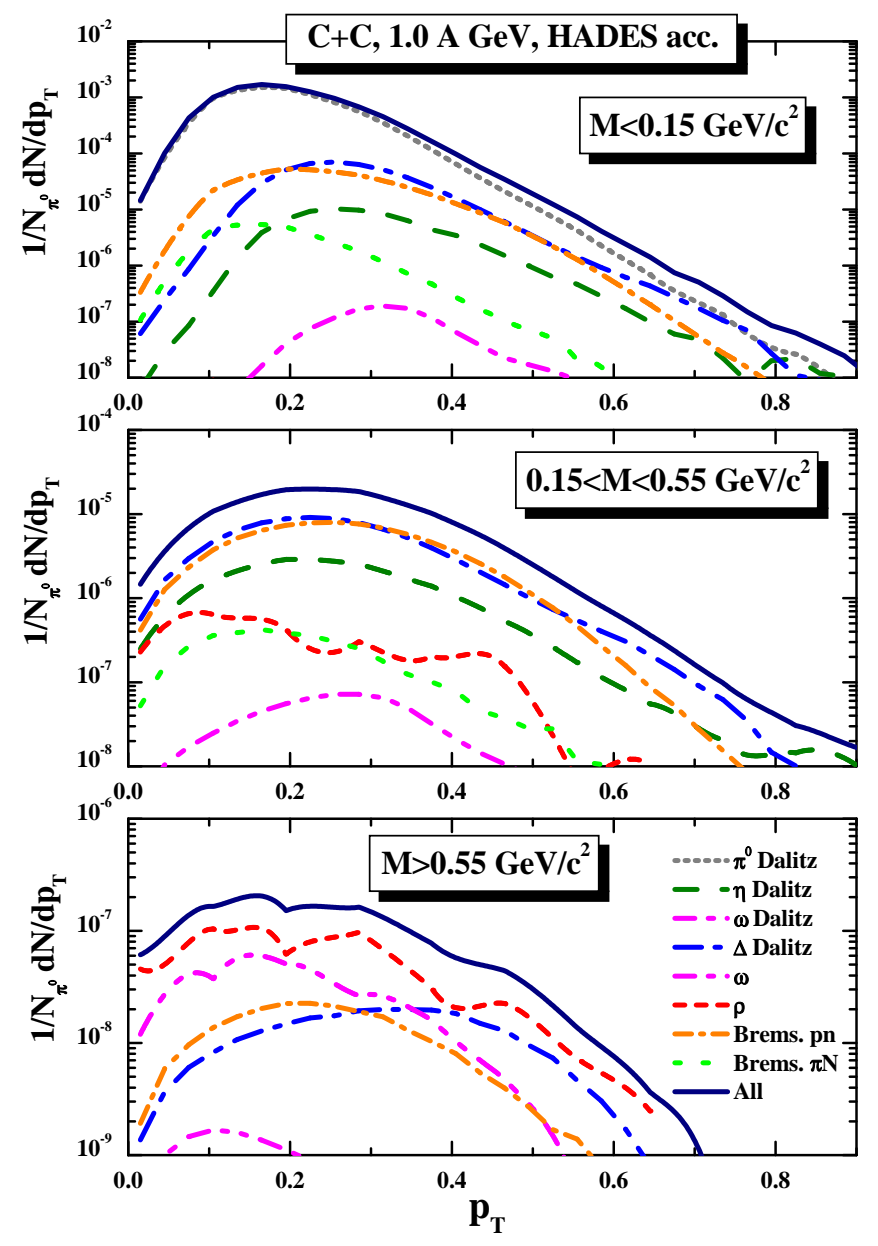

Fig. 19. HSD predictions for the dilepton transverse momentum spectra for ${ }^{12} C+{ }^{12} C$ at $1.0 \mathrm{~A} \cdot \mathrm{GeV}$. The upper part corresponds to the mass range $M \leq 0.15 \mathrm{GeV}$, the middle part to the interval $0.15 \leq M \leq 0.55 \mathrm{GeV}$ and the lower part to $M \geq 0.55 \mathrm{GeV}$. The individual colored lines display the contributions from the various channels in the HSD calculations (see color coding in the legend).

HADES Collaboration is in the process of performing experiments with $p p$ and/or $p d$ reactions at GSI with improved statistics, mass resolution and phase-space coverage. These new experiments will also shed further light on the size of the bremsstrahlung channels in competition with hadron Dalitz decays. In order to prove/disprove the interaction scenarios employed here in HSD we present predictions for the future measurements within the presently known HADES detector acceptance 1 .

We start with elementary reaction channels: Figure 21 shows the differential cross section for $e^{+} e^{-}$production in $p p$ (upper part), $p n$ (middle part) and $p d$ (lower part) reactions at bombarding energies of $1.25 \mathrm{GeV}$ (1.h.s.), $2.2 \mathrm{GeV}$ (middle) and 3.5 $\mathrm{GeV}$ (r.h.s.) including the actual HADES acceptance filter and mass resolution (that

1 Any change in experimental cuts or in the data analysis in future will easily be taken into account since the HSD 'raw data' will be made available to the HADES Collaboration. 


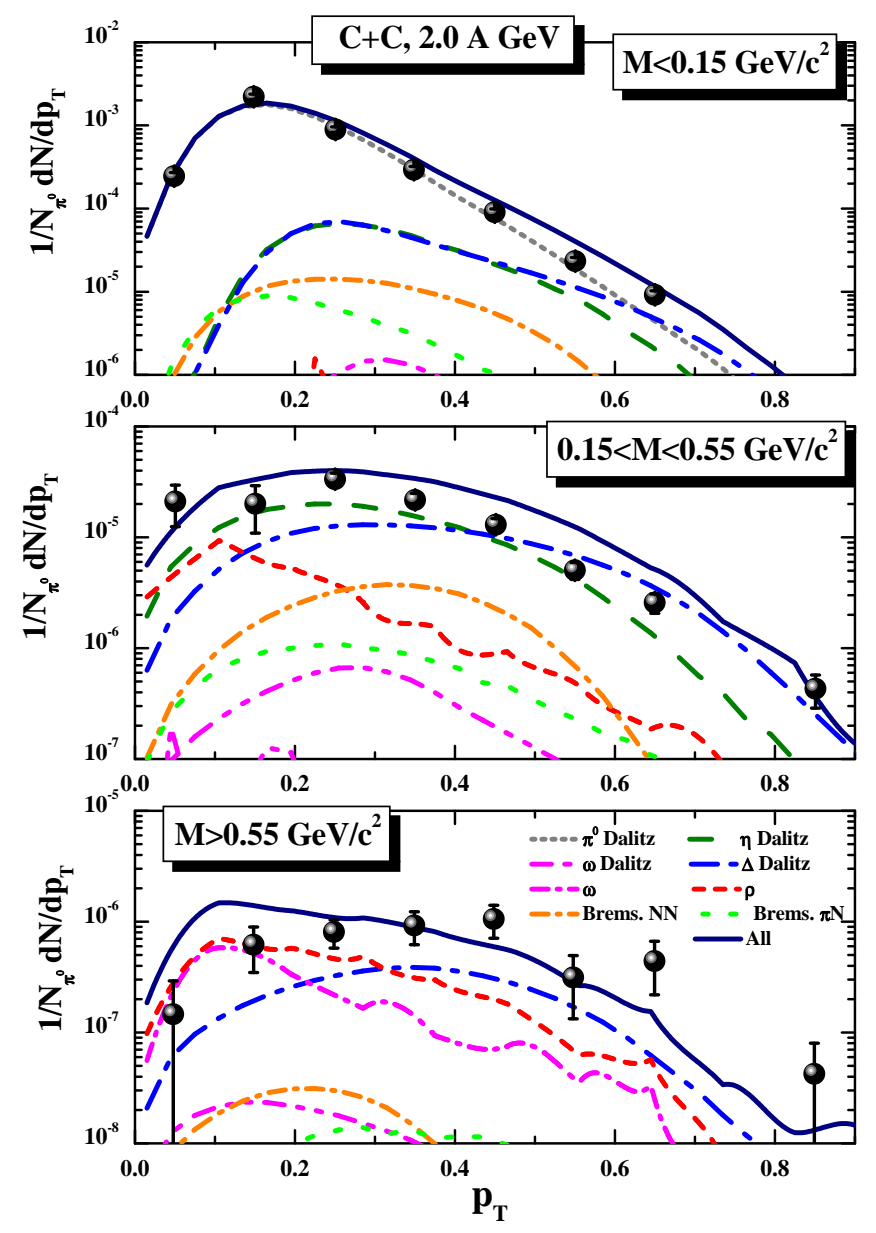

Fig. 20. The dilepton transverse momentum spectra from HSD for ${ }^{12} C+{ }^{12} C$ at $2.0 \mathrm{~A} \cdot \mathrm{GeV}$ in comparison to the data from the HADES Collaboration 62. The upper part corresponds to the mass range $M \leq 0.15 \mathrm{GeV}$, the middle part to the mass range $0.15 \mathrm{GeV} \leq M \leq$ $0.55 \mathrm{GeV}$ and the lower part to $M \geq 0.55 \mathrm{GeV}$. The individual colored lines display the contributions from the various channels in the HSD calculations (see line coding in the legend).

differs in the magnetic settings for the different systems and bombarding energy). We note that the dilepton spectra are normalized to the number of $\pi^{0}$ taken from the HSD calculations and not to $\left(\pi^{+}+\pi^{-}\right) / 2$ as for the heavy-ion reactions. This is important to point out due to the strong isospin asymmetry in $p p, p n$ and $p d$ reactions.

It is seen from Fig. 21 that for low invariant masses $(<0.15 \mathrm{GeV})$ the $\pi^{0}$ Dalitz decays dominates which can be controlled additionally by direct pion measurements. At 1.25 $\mathrm{GeV}$ the $\Delta$ Dalitz decay (dash-dotted blue line) is by far the dominant channel in $p p$ collisions whereas the $N N$ bremsstrahlung channels (dash-dotted orange line) overtake for $p n$ collisions at this energy. In $p d$ reactions the $\Delta$ Dalitz decay and the bremsstrahlung channels contribute with roughly the same magnitude in the entire invariant mass range. The $\eta$ Dalitz decay slightly contributes in the $p d$ case due to 

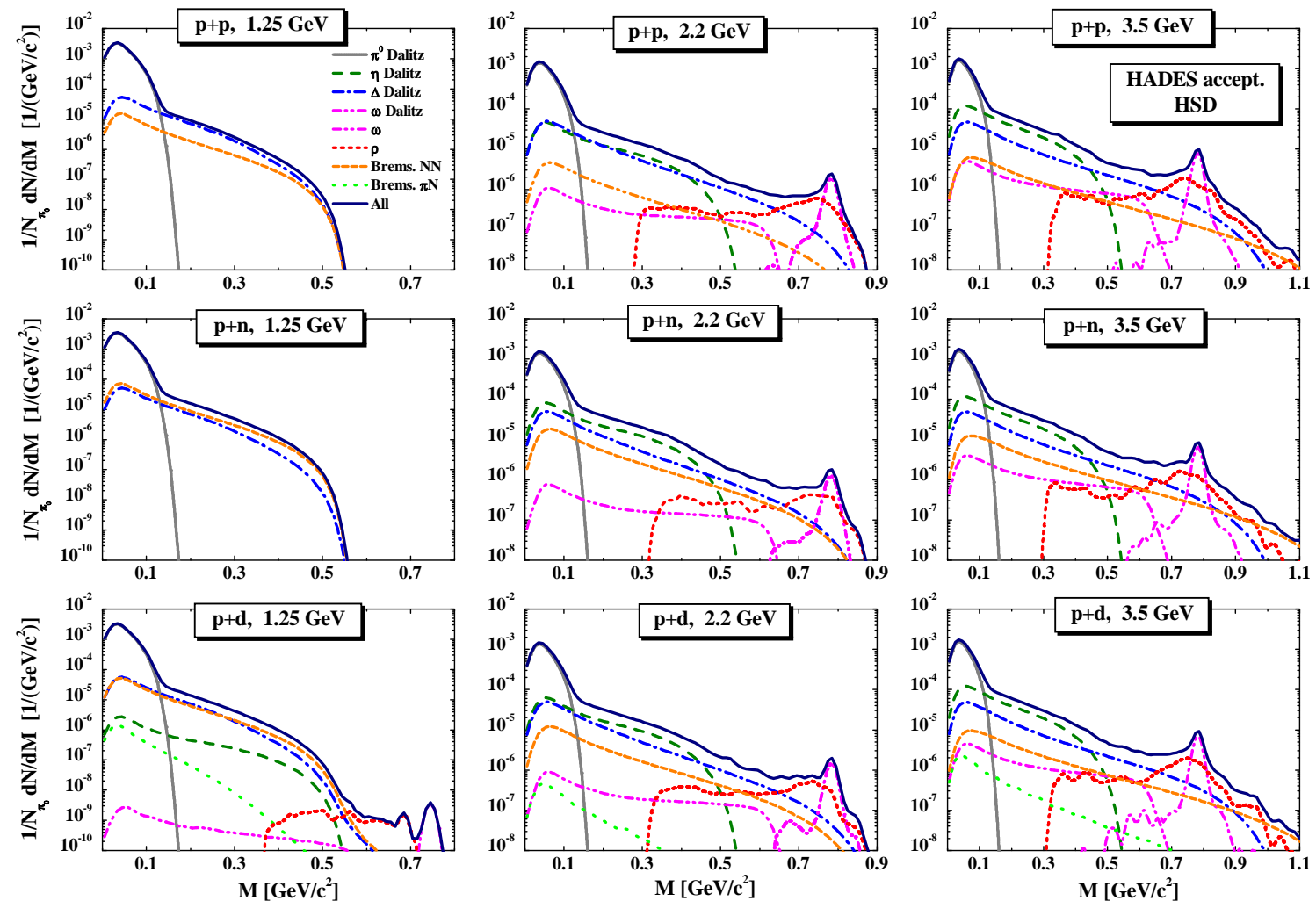

Fig. 21. The differential cross section for $e^{+} e^{-}$production in $p p$ (upper part), $p n$ (middle part) and $p d$ (lower part) reactions at bombarding energies of $1.25 \mathrm{GeV}$ (1.h.s.), $2.2 \mathrm{GeV}$ (middle) and $3.5 \mathrm{GeV}$ (r.h.s.) including the HADES acceptance filter and mass resolution (see legend for the different color coding of the individual channels).

the internal Fermi motion of the nucleons in the deuteron. Discarding the low mass $\pi^{0}$ decays we find a dominance of $\eta$ and $\Delta$ Dalitz decays at 2.2 and $3.5 \mathrm{GeV}$ for $p p, p n$ and $p d$ collisions up to invariant masses of $0.6 \mathrm{GeV}$. Bremsstrahlung channels here are lower by more than an order of magnitude and are hardly to access at this energy. On the other hand the $\pi^{0}, \eta$ and $\Delta$ Dalitz decays may be controlled by direct hadronic measurements. In the vector-meson mass range $(\sim 0.8 \mathrm{GeV})$ the dilepton yield is clearly dominated by the direct $\rho$ and $\omega$ decays; a separation of these channels is possible by measuring additionaly the $\pi^{0} \gamma$ decay channel of the $\omega$ meson as in Ref. [75]. Note that the contribution from pion rescattering on the spectator nucleon in case of $p d$ reactions (dotted light green lines) is down by roughly two orders of magnitude and will be hard to identify experimentally.

We continue with nucleus-nucleus collisions. Since the modifications of the $\rho$-meson spectral function only leave moderate traces in the differential dilepton spectra for ${ }^{12} C+{ }^{12} C$ (cf. Section 5.2) we provide predictions for $\mathrm{Ar}+\mathrm{KCl}$ at $1.75 \mathrm{~A} \cdot \mathrm{GeV}$, a system which is currently analyzed by the HADES Collaboration and might allow for a closer view on the $\rho$-meson properties in a denser medium. The HSD predictions for the mass differential dilepton spectra - divided by the average number of $\left(\pi^{+}+\pi^{-}\right) / 2$ 

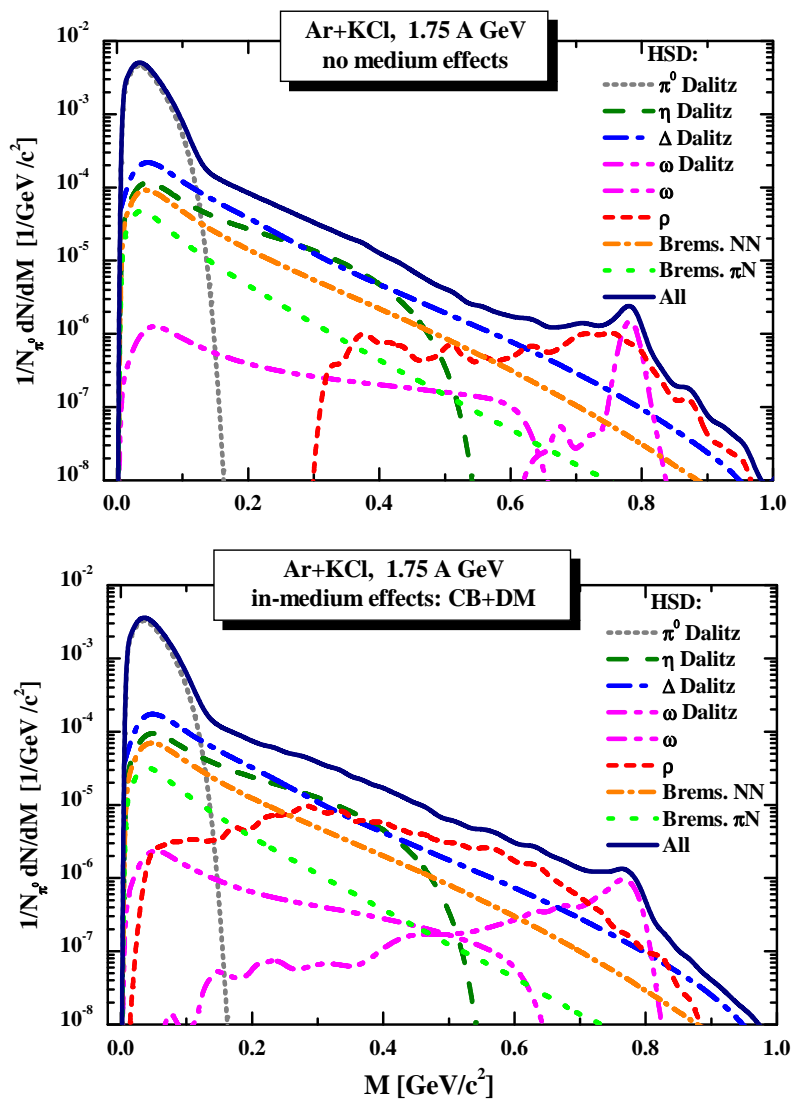

Fig. 22. The HSD predictions for the mass differential dilepton spectra - divided by the average number of $\pi^{0}$ 's taken as $\left(\pi^{+}+\pi^{-}\right) / 2$ - in case of $A r+K C l$ at $1.75 \mathrm{~A} \cdot \mathrm{GeV}$. The upper part shows the case of 'free' vector-meson spectral functions while the lower part gives the result for the 'dropping mass + collisional broadening' scenario. In both scenarios the actual HADES acceptance filter and mass resolution have been incorporated (see legend for the different color coding of the individual channels).

- in this case are presented in Fig. 22. The upper part shows the case of 'free' vectormeson spectral functions while the lower part gives the result for the 'dropping mass + collisional broadening' scenario. In both scenarios the actual HADES acceptance filter and mass resolution have been incorporated. Since the standard Dalitz decays are roughly the same in both scenarios let us concentrate on the direct vector meson decay channels, i.e. the dashed red lines for the $\rho$ meson and the double-dot - dashed purple lines for the $\omega$ meson. The effect of the finite mass resolution can clearly be seen in case of the $\omega$ meson for the 'free' scenario. The peak appears at the pole mass and is dominantly smeared by the experimental mass resolution (except from the moderate vacuum decay width).

In the 'dropping mass + collisional broadening' scenario the shift of the $\omega$ strength to lower invariant masses is clearly seen in the calculations but will be very hard 
to access in experiment. In this respect it might be more favorable to look for the $\pi^{0} \gamma$ decay in comparison to the $e^{+} e^{-}$decay in order to observe experimentally inmedium effects for the $\omega$ meson. In case of the $\rho$ meson the effects of a dropping mass + collisional broadening become clearly visible for invariant masses above 0.3 $\mathrm{GeV}$ resulting in a significant enhancement (by more than a factor of two) in the mass differential dilepton spectra. Actually this in-medium enhancement is slightly lower than predicted in the (more simplified) on-shell quasi-particle approach in the nineties [23,21,44,45] but should be measured experimentally in case of high statistics.

\section{Summary}

In this study a detailed analysis of dilepton production in $C+C$ and $C a+C a$ at 1-2 A. GeV as well as in elementary $p p$ and $p d$ reactions at $1-5 \mathrm{GeV}$ has been presented within the microscopic HSD transport approach that incorporates a full off-shell propagation of the vector mesons in line with Refs. 64,65]. A comparison of dilepton spectra from 'elementary' $p p$ and $p d$ reactions with the DLS data in the energy range from 1 to $4.9 \mathrm{GeV}$ specifies the isospin dependence of the various channels and shows the adequacy/deficiency of the parametrization or model assumptions in the HSD approach.

Our present HSD model includes the following novel developments with respect to the previous studies a decade ago [21,23,44,45]:

- off-shell dynamics for vector mesons and explicit (nonperturbative) propagation of vector mesons also at SIS energies

- improved cross sections for $\eta$ production in $p p$ and $p n$ reactions

- extension of the LUND string model for the production of particles according to 'in-medium' spectral functions

- (enhanced) bremsstrahlung contributions according to Ref. [69] which proofs vital for a solution of the DLS puzzle.

Furthermore, we have analyzed the $\pi^{0}$ and $\eta$ production in $C+C$ collisions at SIS energies. The HSD results for $\pi^{0}$ and $\eta$ productions have been found to be in a good agreement with the data from the TAPS Collaboration from 0.8 to $2 \mathrm{~A} \cdot \mathrm{GeV}$. These studies pin down the dominant contributions from $\pi^{0}$ and $\eta$ Dalitz decays in the low mass dilepton spectra and also to a large extent the contribution from $\Delta$ Dalitz decays which are closely linked to the pion multiplicity.

On the vector meson side different scenarios of in-medium modifications of $\rho$ 's and $\omega$ 's, such as collisional broadening and additionally dropping vector meson masses (cf. Fig. 2), have been investigated and the possibilities for an experimental observation of in-medium effects in $A A$ reactions has been discussed. We find that within the extended off-shell transport approach we achieve a simultaneous description of the differential dilepton spectra for ${ }^{12} \mathrm{C}+{ }^{12} \mathrm{C}$ at $1.04 \mathrm{~A} \cdot \mathrm{GeV}$ from the DLS Collaboration 
as well as the HADES Collaboration which points towards a consistency of the independent measurements within error bars. In general the experimental spectra are better described by the in-medium scenarios for the vector mesons, however, the modifications of the spectra relative to the 'free' scenario are very moderate for the light $C+C$ systems.

In order to shed some light on perspectives for heavier systems we have provided detailed predictions for $\mathrm{Ar}+\mathrm{KCl}$ at $1.75 \mathrm{~A} \cdot \mathrm{GeV}$ as well as elementary $p p$ and $p d$ reactions at $1.25 \mathrm{GeV}, 2.2 \mathrm{GeV}$ and $3.5 \mathrm{GeV}$. The latter systems are addressed also by the HADES Collaboration and in future will shed light on the contribution of bremsstrahlung channels in elastic and inelastic channels at about $1 \mathrm{~A} \cdot \mathrm{GeV}$ and the in-medium modifications of the $\rho$-meson at $1.75 \mathrm{~A} \cdot \mathrm{GeV}$ in the heavier nucleusnucleus system. We stress that new calculations of dilepton bremsstrahlung - based on a consistent full $G-$ (or $T-$ ) matrix approach - are urgently needed from the theoretical side in order to clarify the presently open situation.

\section{Acknowledgement}

The authors acknowledge inspiring discussions with T. Galatyuk, R. Holtzmann, V. Metag, Yv. Pachmayer, P. Salabura, J. Stroth and M. Sudol.

\section{References}

[1] G.E. Brown, M. Rho, Phys. Rev. Lett. 66 (1991) 2720.

[2] T. Hatsuda, S. Lee, Phys. Rev. C46 (1992) R34.

[3] M. Asakawa, C. M. Ko, P. Lévai, X. J. Qiu, Phys. Rev. C46 (1992) R1159.

[4] G. Chanfray, P. Schuck, Nucl. Phys. A545 (1992) 271c.

[5] M. Asakawa, C.M. Ko, Phys. Rev. C48 (1993) R526.

[6] M. Herrmann, B. Friman, W. Nörenberg, Nucl. Phys. A560 (1993) 411.

[7] C.M. Shakin, W.-D. Sun, Phys. Rev. C 49 (1994) 1185.

[8] R. Rapp, G. Chanfray, J. Wambach, Phys. Rev. Lett. 76 (1996) 368.

[9] F. Klingl, W. Weise, Nucl. Phys. A 606 (1996) 329; F. Klingl, N. Kaiser, W. Weise, Nucl. Phys. A624 (1997) 527.

[10] B. Friman, H. J. Pirner, Nucl. Phys. A617 (1997) 496.

[11] R. Rapp, G. Chanfray, J. Wambach, Nucl. Phys. A617 (1997) 472. 
[12] W. Peters, M. Post, H. Lenske, S. Leupold, U. Mosel, Nucl. Phys. A632 (1998) 109; M. Post, S. Leupold, U. Mosel, Nucl. Phys. A689 (2001) 753.

[13] S. Leupold, W. Peters, U. Mosel, Nucl. Phys. A628 (1998) 311.

[14] G. Agakichiev et al., Phys. Rev. Lett. 75 (1995) 1272; Th. Ullrich et al., Nucl. Phys. A610 (1996) 317c; A. Drees, Nucl. Phys. A610 (1996) 536c.

[15] M. A. Mazzoni, Nucl. Phys. A566 (1994) 95c; M. Masera, Nucl. Phys. A590 (1995) 93c; T. Åkesson et al., Z. Phys. C68 (1995) 47.

[16] G. Q. Li, C. M. Ko, G. E. Brown, Phys. Rev. Lett. 75 (1995) 4007.

[17] C. M. Ko, G. Q. Li, G. E. Brown, H. Sorge, Nucl. Phys. A610 (1996) 342c.

[18] W. Cassing, W. Ehehalt, C. M. Ko, Phys. Lett. B363 (1995) 35.

[19] W. Cassing, W. Ehehalt, I. Kralik, Phys. Lett. B377 (1996) 5.

[20] E. L. Bratkovskaya, W. Cassing, Nucl. Phys. A619 (1997) 413.

[21] W. Cassing, E. L. Bratkovskaya, Phys. Rept. 308 (1999) 65.

[22] C. Ernst et al., Phys. Rev. C58 (1998) 447.

[23] W. Cassing, E. L. Bratkovskaya, R. Rapp, J. Wambach, Phys. Rev. C57 (1998) 916.

[24] Y. Kim, R. Rapp, G.E. Brown, M. Rho, Phys. Rev. C 62 (2000) 015202.

[25] R. Rapp, J. Wambach, Adv. Nucl. Phys. 25 (2000) 1.

[26] M. Asakawa, C. M. Ko, Nucl. Phys. A560 (1993) 399.

[27] G. Q. Li, C. M. Ko, G. E. Brown, Nucl. Phys. A606 (1996) 568.

[28] R. Arnaldi et al., Phys. Rev. Lett. 96 (2006) 162302; J. Seixas et al., J. Phys. G 34 (2007) S1023.

[29] G. Agakichiev et al., Eur. Phys. J. C 41 (2005) 475.

[30] R. Rapp, J. Phys. G 34 (2007) S405.

[31] R. Rapp, Nucl. Phys. A 782 (2007) 275.

[32] H. van Hees, R. Rapp, Phys. Rev. Lett. 97 (2006) 102301.

[33] J. Ruppert, C. Gale, T. Renk, P. Lichard, J. I. Kapusta, e-Print: arXiv:0706.1934 [hep-ph]

[34] C. Gale, S. Turbide, Nucl. Phys. A 783 (2007) 351.

[35] J. Ruppert, T. Renk, Phys. Rev. C 71 (2005) 064903; Erratum-ibid. C 75 (2007) 059901; T. Renk, J. Ruppert, hep-ph/0605130.

[36] T. Renk, R. A. Schneider, W. Weise, Phys. Rev. C 66 (2002) 014902.

[37] K. Dusling, D. Teaney, I. Zahed, Phys. Rev. C 75 (2007) 024908; K. Dusling, I. Zahed, arXiv:0712.1982 [nucl-th]. 
[38] G. Roche et al., DLS Collaboration, Phys. Rev. Lett. 61 (1988) 1069; C. Naudet et al., Phys. Rev. Lett. 62 (1989) 2652; G. Roche et al., Phys. Lett. B226 (1989) 228.

[39] R.J. Porter et al., DLS Collaboration, Phys. Rev. Lett. 79 (1997) 1229.

[40] L. Xiong, Z. G. Wu, C. M. Ko, J. Q. Wu, Nucl. Phys. A 512 (1990) 772.

[41] Gy. Wolf et al., Nucl. Phys. A517 (1990) 615; Gy. Wolf, W. Cassing, U. Mosel, Nucl. Phys. A552 (1993) 549.

[42] K.K. Gudima, A.I. Titov, V.D. Toneev, Sov. Jour. of Nucl. Phys. 55 (1992) 1715.

[43] E. L. Bratkovskaya, W. Cassing, U. Mosel, Phys. Lett. B376 (1996) 12.

[44] E. L. Bratkovskaya, W. Cassing, R. Rapp, J. Wambach, Nucl. Phys. A634 (1998) 168.

[45] E. L. Bratkovskaya, C. M. Ko, Phys. Lett. B445 (1999) 265.

[46] J.H. Koch, E.J. Moniz, and N. Ohtsuka, Ann.of Phys. 154 (1984) 99.

[47] K. Shekhter, C. Fuchs et al., Phys. Rev. C68 (2003) 014904.

[48] E. L. Bratkovskaya, Nucl. Phys. A696 (2001) 761.

[49] E. L. Bratkovskaya, Phys. Lett. B529 (2002) 26.

[50] D. Schumacher, S. Vogel, M. Bleicher, Acta Phys. Hung. A 27 (2006) 451.

[51] S. Vogel, H. Petersen, J. Aichelin, and M. Bleicher, arXiv:0710.4463 [hep-ph].

[52] M. Effenberger, E. L. Bratkovskaya, W. Cassing, U. Mosel, Phys. Rev. C 60 (1999) 027601.

[53] A. Fässler, C. Fuchs, Int. J. Mod. Phys. E 16 (2007) 879.

[54] M.D. Cozma, C. Fuchs, E. Santini, A. Fässler, Phys. Lett. B 640 (2006) 170.

[55] C. Fuchs, A. Fässler, Prog. Part. Nucl. Phys. 53 (2004) 59.

[56] M. Thomere, C. Hartnack, Gy. Wolf, J. Aichelin, Phys. Rev. C 75 (2007) 064902.

[57] H. W. Barz, B. Kämpfer, Gy. Wolf, M. Zetenyi, e-Print: nucl-th/0605036 .

[58] K. Weber et al., Nucl. Phys. A 539 (1992) 713.

[59] T. Falter et al., Phys. Lett. B 594 (2004) 61; Phys. Rev. C 70 (2004) 054609.

[60] J. Pietraszko et al., HADES Collaboration, Int. J. Mod. Phys. A 22 (2007) 388.

[61] G. Agakichiev et al., HADES Collaboration, Phys. Rev. Lett. 98 (2007) 052302.

[62] T. Eberl et al., HADES Collaboration, Eur. Phys. J. C 49 (2007) 261.

[63] G. Agakishiev et al., HADES Collaboration, arXiv:0711.4281 [nucl-ex].

[64] W. Cassing, S. Juchem, Nucl. Phys. A 665 (2000) 377. 
[65] W. Cassing, S. Juchem, Nucl. Phys. A 672 (2000) 417.

[66] W. Ehehalt, W. Cassing, Nucl. Phys. A 602 (1996) 449.

[67] B. Anderson, G. Gustafson, Hong Pi, Z. Phys. C 57 (1993) 485.

[68] E.L. Bratkovskaya, W. Cassing, U. Mosel, Nucl. Phys. A686 (2001) 568.

[69] L.P. Kaptari, B. Kämpfer, Nucl. Phys. A 764 (2006) 338.

[70] I. Fröhlich et al., arXiv:0708.2382 [nucl-ex].

[71] G. Q. Li, C. M. Ko, Nucl. Phys. A 582 (1995) 731.

[72] W. Cassing, Ye. S. Golubeva, A. S. Iljinov, L. A. Kondratyuk, Phys. Lett. B 396 (1997) 26; Ye. S. Golubeva, L. A. Kondratyuk, W. Cassing, Nucl. Phys. A625 (1997) 832.

[73] L. M. Barkov et al., Nucl. Phys. B 256 (1985) 365.

[74] V. Metag, arXiv:0711.4709 [nucl-ex].

[75] D. Trnka et al., Phys. Rev. Lett. 94 (2005) 192303.

[76] A. Sibirtsev, W. Cassing, Nucl. Phys. A 641 1998) 476.

[77] Landolt-Börnstein, New Series, ed. H. Schopper, I/12 (1988).

[78] F. Balestra et al., Phys. Rev. C 63 (2001) 024004; Phys. Rev. Lett. 89 (2002) 092001.

[79] C. Gale, J. Kapusta, Phys. Rev. C 35 (1987) 2107; C. Gale, J. Kapusta, Nucl. Phys. A 495 (1989) 423c.

[80] P. Lichard, Phys. Rev. D 51 (1995) 6017.

[81] M. Schäfer, T. S. Biro, W. Cassing, U. Mosel, Phys. Lett. B 221 (1989) 1.

[82] R. Shyam, U. Mosel, Phys. Rev. C 67 (2003) 065202.

[83] F. de Jong, U. Mosel, Phys. Lett. B 392 (1997) 273.

[84] S. Kondratyuk, O. Scholten, Nucl. Phys. A 677 (2000) 396; Phys. Rev. C 62 (2000) 025203 .

[85] R. Holzmann et al., Phys. Rev. C 56 (1997) R2920.

[86] E. L. Bratkovskaya, W. Cassing, U. Mosel, Phys. Lett. B 424 (1998) 244.

[87] H. Calén et al., Phys. Rev. C 58 (1998) 2667.

[88] P. Moskal et al., Prog. Part. Nucl. Phys. 49 (2002) 1;

[89] P. Moskal et al., Int. J. Mod. Phys. A 20 (2005) 1880.

[90] K. Tsushima, A. Sibirtsev, A. W. Thomas, Phys. Rev. C 59 (1999) 369; Erratum-ibid. C 61 (2000) 029903.

[91] R. Averbeck et al., TAPS Collaboration, Z. Phys. A 359 (1997) 65. 
[92] W.K. Wilson et al., DLS Collaboration, Phys. Rev. C 57 (1998) 1865.

[93] M. Effenberger, E. L. Bratkovskaya, U. Mosel, Phys. Rev. C 60 (1999) 044614.

[94] M. Zétényi and Gy. Wolf, Heavy Ion Phys. 17 (2003) 27; Phys. Rev. C 67 (2003) 044002. 